\title{
Certain bivariate distributions and random processes connected with maxima and minima
}

\author{
Tomasz J. Kozubowski ${ }^{1}$ Krzysztof Podgórski ${ }^{2}$
}

Received: 5 June 2017 / Revised: 2 February 2018 / Accepted: 5 February 2018/

Published online: 17 February 2018

(C) The Author(s) 2018. This article is an open access publication

\begin{abstract}
The minimum and the maximum of $t$ independent, identically distributed random variables have $\bar{F}^{t}$ and $F^{t}$ for their survival (minimum) and the distribution (maximum) functions, where $\bar{F}=1-F$ and $F$ are their common survival and distribution functions, respectively. We provide stochastic interpretation for these survival and distribution functions for the case when $t>0$ is no longer an integer. A new bivariate model with these margins involve maxima and minima with a random number of terms. Our construction leads to a bivariate max-min process with $t$ as its time argument. The second coordinate of the process resembles the well-known extremal process and shares with it the one-dimensional distribution given by $F^{t}$. However, it is shown that the two processes are different. Some fundamental properties of the max-min process are presented, including a distributional Markovian characterization of its jumps and their locations.
\end{abstract}

Keywords Copula $\cdot$ Distribution theory $\cdot$ Exponentiated distribution · Extremal process · Extremes · Generalized exponential distribution · Order statistics ·

Random minimum · Random maximum - Sibuya distribution · Pareto distribution . Fréchet distribution

AMS 2010 Subject Classification Primary-60G70; Secondary-60E05

Krzysztof Podgórski

Krzysztof.Podgorski@stat.lu.se

1 Department of Mathematics \& Statistics, University of Nevada, Reno, NV 89557, USA

2 Department of Statistics, Lund University, Lund, Sweden 


\section{Introduction}

For a sequence of independent and identically distributed (IID) random variables $\left\{X_{i}\right\}$ with cumulative distribution function (CDF) $F$ and survival function (SF) $\bar{F}=$ $1-F$, the extreme order statistics are the minimum

$$
S_{n}=\bigwedge_{j=1}^{n} X_{j}=\min \left\{X_{1}, \ldots, X_{n}\right\}
$$

and the maximum

$$
M_{n}=\bigvee_{j=1}^{n} X_{j}=\max \left\{X_{1}, \ldots, X_{n}\right\}
$$

These are crucial in countless applications and became the central and well-studied objects of extreme value theory (see, e.g., monographs Beirlant et al. 1996; Castillo 1988; Embrechts et al. 1997; Galambos 1987; Gumbel 1958; Kotz and Nadarajah 2000; Leadbetter et al. 1983; Reiss and Thomas 2001; Resnick 1987 and extensive references therein as well as in Harter 1978). It is well-known (see, e.g, Arnold et al. 1992 or David 1981) that the SF of $S_{n}$ and the CDF of $M_{n}$ are given by $\bar{F}^{n}$ and $F^{n}$, respectively, while the joint $\mathrm{CDF}$ of the vector $\left(S_{n}, M_{n}\right)$ has the form

$$
\mathbb{P}\left(S_{n} \leq x, M_{n} \leq y\right)= \begin{cases}F^{n}(y)-(F(y)-F(x))^{n} & \text { for } x<y, \\ F^{n}(y) & \text { for } x \geq y .\end{cases}
$$

It is rather obvious that the functions

$$
\bar{F}^{\alpha}(x)=(\bar{F}(x))^{\alpha}, F^{\alpha}(x)=(F(x))^{\alpha}
$$

are genuine survival and distribution functions, respectively, for any positive value of $\alpha$, although simple interpretations are no longer available for non-integer $\alpha$. Nevertheless, many interesting, flexible, and useful univariate families of distributions have been defined this way over the years, with earliest works connected with an exponential model going back to the first half of the nineteenth century (see, e.g., Gompertz 1825). In addition to the exponential, these new classes of distributions - often referred to as exponentiated distributions - include those connected with gamma, Pareto, and Weibull laws (see, e.g., Gupta et al. 1998; Gupta and Kundu 1999, 2007; Mudholkar and Srivastava 1993; Mudholkar et al. 1995; Pal et al. 2006 and references therein).

In this work we study a bivariate generalization of the joint distribution (1.3) in the same spirit, where the margins are given by Eq. 1.4 with $\alpha>0$, and the bivariate model reduces to Eq. 1.3 when $\alpha$ is a non-negative integer. Moreover, the bivariate model with a positive non-integer $\alpha$, as well as the margins (1.4), are constructed through maxima and minima. Such a generalization is not obvious and cannot arise, for example, by simply replacing the integer $n$ by $\alpha$ in the bivariate CDF (1.3) (the latter generally fails to be a CDF when $\alpha \in(0,1))$. Instead, the model follows a construction involving random maxima and minima of IID random variables, naturally extending the connection between $\bar{F}^{n}$ and $F^{n}$ and the distribution of min-max variables $\left(S_{n}, M_{n}\right)$. 
Our approach leads to a bivariate stochastic process $(X(t), Y(t)), t>0$, whose one dimensional marginals are given by Eq. 1.4 with $\alpha=t$. This new process can be viewed as a (bivariate) generalization of the classical concept of the extremal process, introduced in Dwass $(1964,1966)$ and Lamperti (1964). The latter expresses in terms of a pure jump non-decreasing Lévy process given by a sequence of pairs $\left(H_{i}, \tau_{i}\right)$ representing jump sizes and their location as

$$
M(t)=\max \left\{H_{i}, \tau_{i} \leq t\right\}, \quad t>0 .
$$

As shown in the sequel, the extremal process $M(t)$, which has the same onedimensional marginal distributions $F^{t}$, shares some structural properties with $Y(t)$ as well, but the two processes are essentially different.

Our work begins with Section 2, which introduces new stochastic representations for random variables given by Eq. 1.4 through random maxima and minima. These lead to new representations of the variables with exponentiated distributions based on exponential, gamma, Weibull, Pareto and other models. These results are instrumental in defining a bivariate model presented in Section 3 and the concept of duality, discussed in Section 4. Generalizations to random process are presented in Section 5, where the case of the unit interval is discussed, and in Section 6, where the discussion is extended to the positive half-line. The results in these two sections include an account of similarities and differences between the newly introduced processes and the extremal process. The last section contains proofs and auxiliary results.

\section{Univariate distributions}

Let $\left\{X_{i}\right\}$ be a sequence of IID random variables with CDF $F$ and SF $\bar{F}$, and let $N$ be an $\mathbb{N}$-valued random variable, where $\mathbb{N}=\{1,2, \ldots\}$ is the set of natural numbers. Further, we let $p(n)=\mathbb{P}(N=n)$ and $G_{N}(s)=\mathbb{E} s^{N},|s| \leq 1$, be the probability density function (PDF) and the probability generating function (PGF) of $N$, respectively. The focus of our work is on representing random variables with the SF or the CDF given in Eq. 1.4 through maxima and minima of a random number of independent and identically distributed random variables. The following basic lemma is crucial in obtaining such representations and its elementary proof is given in the Appendix.

Lemma 2.1 If an $\mathbb{N}$-valued random variable $N$ is independent of the sequence $\left\{X_{i}\right\}$, then the $C D F$ and the $S F$ of the random variables

$$
X=\bigvee_{j=1}^{N} X_{j} \text { and } Y=\bigwedge_{j=1}^{N} X_{j}
$$

are given by $F_{X}(x)=G_{N}(F(x))$ and $\bar{F}_{Y}(y)=G_{N}(\bar{F}(y))$, respectively.

We shall use this result to obtain representations of random variables with the SF and the CDF given in Eq. 1.4 through random maxima and minima with suitably 
chosen $N$. This generally cannot be accomplished by insisting that the CDF of $X$ be $F^{\alpha}$ and the SF of $Y$ be $\bar{F}^{\alpha}$. Indeed, no PGF will satisfy

$$
G_{N}(F(x))=F^{\alpha}(x), \quad G_{N}(\bar{F}(y))=\bar{F}^{\alpha}(x),
$$

for each $\operatorname{CDF} F$ and SF $\bar{F}$, unless $\alpha$ is an integer, since it would be equivalent to $G_{N}(s)=s^{\alpha}$ leading to $N=\alpha$.

To obtain a solution to this problem, one has to swap the maximum with the minimum, and instead stipulate that the SF of $X$ be $\bar{F}^{\alpha}$ and the CDF of $Y$ be $F^{\alpha}$. According to Lemma 2.1, this leads to the equations

$$
1-G_{N}(F(x))=\bar{F}^{\alpha}(x), 1-G_{N}(\bar{F}(y))=F^{\alpha}(y),
$$

which are equivalent to

$$
G_{N}(s)=1-(1-s)^{\alpha}, \quad|s| \leq 1
$$

The above expression is indeed a genuine PGF, albeit only for $\alpha \in(0,1]$, and represents a Sibuya random variable $N_{\alpha}$ (see, Sibuya 1979) with the PDF

$$
p_{\alpha}(n)=\mathbb{P}\left(N_{\alpha}=n\right)=\left(\begin{array}{l}
\alpha \\
n
\end{array}\right)(-1)^{n+1}=\frac{\alpha(\alpha-1) \cdots(\alpha-n+1)}{n !}(-1)^{n+1}, \quad n \in \mathbb{N},
$$

arising in connection with discrete stable, Linnik, and Mittag-Leffler distributions (see, e.g., Christoph and Schreiber 1998, 2000; Devroye 1993; Pakes 1995; Pillai and Jayakumar 1995).

Remark 2.2 Expressing the function (2.2) as a power series,

$$
1-(1-s)^{\alpha}=\sum_{n=1}^{\infty}\left(\begin{array}{l}
\alpha \\
n
\end{array}\right)(-1)^{n+1} s^{n}, \quad|s| \leq 1,
$$

makes it clear that this is not a PGF when $\alpha>1$, as then not all coefficients of $s^{n}$ in the above series are non-negative (alternatively, the function in (2.4) is not convex in this case). For $\alpha \in(0,1]$ this variable represents the number of trials till the first success in an infinite sequence of independent Bernoulli trials where the $n$th trial is a success with probability $\alpha / n$. Clearly, at the boundary value $\alpha=1$, the distribution degenerates to an atomic measure with a single atom at $n=1$ (Dirac measure). Let us also mention that in the special case $\alpha=1 / 2$, we obtain the distribution of $Z+1$, where $Z$ has a discrete Mittag-Leffler distribution with the PGF $G(s)=$ $\left(1+c(1-s)^{\alpha}\right)^{-1}$ with $c=1$ (see, e.g., Pillai and Jayakumar 1995).

In view of this discussion, we obtain the following result.

Corollary 2.3 Let $F$ be a distribution function on $\mathbb{R}$ and $\bar{F}$ be the corresponding survival function, $\bar{F}(x)=1-F(x)$. Further, let $X$ and $Y$ have SF and CDF given 
by $\bar{F}^{\alpha}$ and $F^{\alpha}$, respectively, where $\alpha \in(0,1]$. Then $X$ and $Y$ admit the stochastic representations

$$
X \stackrel{d}{=} \bigvee_{j=1}^{N_{\alpha}} X_{j} \text { and } Y \stackrel{d}{=} \bigwedge_{j=1}^{N_{\alpha}} X_{j}
$$

where $N_{\alpha}$ has the Sibuya distribution (2.3) and is independent of the IID $\left\{X_{j}\right\}$ with the CDF F.

Remark 2.4 Let us note that if the equalities in distribution in Eq. 2.5 were replaced by actual equalities, then the variables $X$ and $Y$ from Corollary 2.3 would satisfy the relation $X \geq Y$. The latter inequality between random variables induces stochastic ordering between their distributions,

$$
\mathbb{P}(Y>x)=1-F^{\alpha}(x) \leq(1-F(x))^{\alpha}=\mathbb{P}(X>x), \quad x \in \mathbb{R},
$$

which holds for the case $\alpha \leq 1$. This property, also known as the first order stochastic dominance, reversely extends to the case of $\alpha>1$. Namely, by replacing $F$ in the above by $F^{1 / \alpha}$, we obtain

$$
1-F(x) \leq\left(1-F^{1 / \alpha}(x)\right)^{\alpha},
$$

which is equivalent to

$$
1-F^{\alpha^{\prime}}(x) \geq(1-F(x))^{\alpha^{\prime}}, x \in \mathbb{R},
$$

for $\alpha^{\prime}=1 / \alpha \in(1, \infty)$.

An extension of Corollary 2.3 to the case $\alpha \in(0, \infty)$ is rather straightforward. In the following result and throughout the paper we shall use the notation:

$$
\left[t^{-}\right]=k \text { and }\langle t\rangle=t-k \text { whenever } k<t \leq k+1, k=0,1,2, \ldots
$$

Note that the quantity $\left[t^{-}\right]$is the same as the standard integer part of $t$, commonly denoted by $[t]$, whenever $t$ is not an integer. However, $\left[n^{-}\right]=n-1$ while $[n]=n$ for $n \in \mathbb{N}$. Here, the min and the max over an empty set are understood as $\infty$ and $-\infty$, respectively.

Corollary 2.5 Let $F$ be a distribution function on $\mathbb{R}$ and $\bar{F}$ be the corresponding survival function, $\bar{F}(x)=1-F(x)$. Further, let $X$ and $Y$ have SF and CDF given by $\bar{F}^{t}$ and $F^{t}$, respectively, where $t \in(0, \infty)$. Then $X$ and $Y$ admit the stochastic representations

$$
X \stackrel{d}{=} \bigwedge_{j=1}^{\left[t^{-}\right]} X_{j} \wedge \bigvee_{j=\left[t^{-}\right]+1}^{\left[t^{-}\right]+N_{\langle t\rangle}} X_{j} \text { and } Y \stackrel{d}{=} \bigvee_{j=1}^{\left[t^{-}\right]} X_{j} \vee \bigwedge_{j=\left[t^{-}\right]+1}^{\left[t^{-}\right]+N_{\langle t\rangle}} X_{j}
$$

where $N_{\langle t\rangle}$ has the Sibuya distribution (2.3) with parameter $\alpha=\langle t\rangle$ and is independent of the IID $\left\{X_{j}\right\}$ with the CDF F. 


\section{Bivariate models}

An extension to bivariate distribution having margins with SF and CDF given by Eq. 1.4 is straightforward. One such distribution is obtained by putting together the $X$ and $Y$ defined in Corollary 2.3. Let us start with the following result, which extends Lemma 2.1 to the bivariate setting.

\section{Lemma 3.1 Let}

$$
(X, Y)=\left(\bigvee_{j=1}^{N} X_{j}, \bigwedge_{j=1}^{N} X_{j}\right),
$$

where the $\left\{X_{i}\right\}$ are IID random variables, independent of an $\mathbb{N}$-valued random variable $N$. Then, the CDF of $(X, Y)$ is given by

$$
H(x, y)= \begin{cases}G_{N}(F(x))-G_{N}(F(x)-F(y)) & \text { for } x>y \\ G_{N}(F(x)) & \text { for } x \leq y,\end{cases}
$$

where $G_{N}(\cdot)$ and $F(\cdot)$ are the PGF of $N$ and the common CDF of the $\left\{X_{i}\right\}$, respectively.

According to Lemma 2.1, the marginal CDF of $X$ in Eq. 3.1 is $F_{X}(x)=$ $G_{N}(F(x))$ while the marginal SF of $Y$ in Eq. 3.1 $\bar{F}_{Y}(y)=G_{N}(\bar{F}(y))$, where $\bar{F}(y)=1-F(y)$ is the common SF of the $\left\{X_{i}\right\}$. In particular, by taking $N$ to be Sibuya distributed $N_{\alpha}$ given by the PGF (2.2), we obtain a bivariate distribution with marginal SF of $X$ and the marginal CDF of $Y$ given by $\bar{F}^{\alpha}$ and $F^{\alpha}$, respectively. The following result summarizes this.

\section{Corollary 3.2 Let}

$$
(X, Y) \stackrel{d}{=}\left(\bigvee_{j=1}^{N_{\alpha}} X_{j}, \bigwedge_{j=1}^{N_{\alpha}} X_{j}\right),
$$

where $N_{\alpha}$ is Sibuya distributed given by the PGF (2.2) with $\alpha \in(0,1]$ and the $\left\{X_{i}\right\}$ are IID random variables, independent of $N_{\alpha}$. Then, the joint $C D F$ of $(X, Y)$ is given by

$$
H(x, y)= \begin{cases}(1-F(x)+F(y))^{\alpha}-(1-F(x))^{\alpha} & \text { for } x>y \\ 1-(1-F(x))^{\alpha} & \text { for } x \leq y\end{cases}
$$

the marginal SF of $X$ is $\bar{F}_{X}=\bar{F}^{\alpha}$, while the marginal $C D F$ of $Y$ is $F_{Y}=F^{\alpha}$, where $\bar{F}$ and $F$ are the common $S F$ and $C D F$ of the $\left\{X_{i}\right\}$, respectively.

Remark 3.3 In the special case $\alpha=1$, the joint CDF (3.4) becomes

$$
S(x, y)=\left\{\begin{array}{l}
F(y) \text { for } x>y, \\
F(x) \text { for } x \leq y,
\end{array}\right.
$$

and it describes the random vector $\left(X_{1}, X_{1}\right)$, to which Eq. 3.3 reduces in this case (since $N_{\alpha}=1$ when $\alpha=1$ ). Note that this CDF is also a special case $n=1$ of the joint CDF of the two extreme order statistics, $S_{n}$ and $M_{n}$, given by Eq. 1.3. 
Note that if the distribution of the underlying random variables $\left\{X_{i}\right\}$ is absolutely continuous, the bivariate $\mathrm{CDF} H$ in the above results is a continuous function. However, the bivariate distribution defined viz. $H$ may fail to be absolutely continuous. Indeed, whenever $p(1)=\mathbb{P}(N=1)>0$, the probability measure induced by $H$ in Eq. 3.2 has a singular part supported on the curve $y=x$. The following result, whose proof is routine, provides further details for the case when $N$ is Sibuya distributed.

Proposition 3.4 Suppose that the $\left\{X_{i}\right\}$ in Eq. 3.3 have absolutely continuous distribution with $C D F F$ and PDF $f$. Then, the CDF $H$ of the random vector $(X, Y)$ in Eq. 3.3 admits the representation

$$
H(x, y)=(1-\alpha) G(x, y)+\alpha S(x, y), \quad x, y \in \mathbb{R},
$$

where

$$
G(x, y)=\frac{1}{1-\alpha} \begin{cases}(1-F(x)+F(y))^{\alpha}-(1-F(x))^{\alpha}-\alpha F(y) & \text { for } x>y, \\ 1-(1-F(x))^{\alpha}-\alpha F(x) & \text { for } x \leq y,\end{cases}
$$

is the CDF of an absolutely continuous bivariate distribution with the PDF

$$
g(x, y)= \begin{cases}\alpha f(x) f(y)(1-F(x)+F(y))^{\alpha-2} & \text { for } x>y, \\ 0 & \text { for } x \leq y,\end{cases}
$$

and $S(x, y)$ is the CDF of the random vector $\left(X_{1}, X_{1}\right)$ given by Eq. 3.5.

Remark 3.5 We note that the $\operatorname{CDF} G(x, y)$ in Eq. 3.7 corresponds to the random vector $(\hat{X}, \hat{Y})$ defined by the right-hand-side of Eq. 3.3 but with $N_{\alpha}$ replaced by its truncated version, $\hat{N}_{\alpha}=N_{\alpha} \mid N_{\alpha}>1$. A representation similar to Eq. 3.6 holds for a general $\mathbb{N}$-distributed $N$ as well as long as $p(1)=\mathbb{P}(N=1) \in[0,1)$. In this case the weights $1-\alpha, \alpha$ are replaced by $1-p(1), p(1)$, the singular $\operatorname{CDF} S(x, y)$ is the same, and $G(x, y)$ is the CDF of the random vector in Eq. 3.1 but with $N$ replaced with $\hat{N} \stackrel{d}{=} N \mid N>1$. That CDF is provided by Lemma 3.1 with $G_{N}$ replaced by the PGF of $\hat{N}$, given by

$$
G_{\hat{N}}(s)=\frac{G_{N}(s)-s p(1)}{1-p(1)} .
$$

Further, the corresponding marginal distributions are provided by Lemma 2.1 with $G_{N}$ replaced by the above $G_{\hat{N}}$. In particular, it can be shown that in the Sibuya case discussed in Proposition 3.4, the marginal SF of $\hat{X}$ (connected with the first coordinate of $G(x, y))$ is given by $\bar{F}_{\hat{X}}(x)=W_{\alpha}(\bar{F}(x))$ while the CDF of $\hat{Y}$ (connected with the second coordinate of $G(x, y))$ is given by $F_{\hat{Y}}(y)=W_{\alpha}(F(y))$, where

$$
W_{\alpha}(u)=\frac{u^{\alpha}-\alpha u}{1-\alpha}, \quad u \in[0,1], \alpha \in(0,1),
$$

and $F(\cdot), \bar{F}(\cdot)$ are the common CDF and SF of the $\left\{X_{i}\right\}$.

Observe that replacing $n$ in the joint CDF (1.3) of the two extreme order statistics with $\alpha \in(0,1)$ does not produce the CDF (3.4). Moreover, the resulting quantity is not even a proper CDF, as can be easily verified. Our next result provides a generalization of the bivariate $\operatorname{CDF}(1.3)$ to non-integer values of $n$. 
Proposition 3.6 Let $t \in(0, \infty)$ and write $t=\left[t^{-}\right]+\langle t\rangle$ with $\left[t^{-}\right] \in \mathbb{N}_{0}=\{0\} \cup \mathbb{N}$ and $\langle t\rangle \in(0,1]$, as in Eq. 2.6. Then the joint CDF of

$$
(X, Y) \stackrel{d}{=}\left(\bigwedge_{j=1}^{\left[t^{-}\right]} X_{j} \wedge \bigvee_{j=\left[t^{-}\right]+1}^{\left[t^{-}\right]+N_{\langle t\rangle}} X_{j}, \bigvee_{j=1}^{\left[t^{-}\right]} X_{j} \vee \bigwedge_{j=\left[t^{-}\right]+1}^{\left[t^{-}\right]+N_{\langle t\rangle}} X_{j}\right)
$$

where $N_{\langle t\rangle}$ has the Sibuya distribution (2.3) with $\alpha=\langle t\rangle$, and is independent of the IID $\left\{X_{j}\right\}$ with the CDF $F$ and the $S F \bar{F}$, is given by

$$
H(x, y)= \begin{cases}F^{t}(y)-(F(y)-F(x))^{\left[t^{-}\right]}\left(F^{\langle t\rangle}(y)-1+\bar{F}^{\langle t\rangle}(x)\right) & \text { for } x<y, \\ F^{t}(y)-\left(F^{\langle t\rangle}(y)-(\bar{F}(x)+F(y))^{\langle t\rangle}+\bar{F}^{\langle t\rangle}(x)\right) \mathbb{I}_{\{0\}}\left(\left[t^{-}\right]\right) & \text {for } x \geq y,\end{cases}
$$

where $\mathbb{I}_{A}(\cdot)$ is the indicator function of a set $A$.

Remark 3.7 Note that, by Corollary 2.5, the marginal SF of $X$ is $\bar{F}^{t}$ and the marginal CDF of $Y$ is $F^{t}$. This can also be seen by taking the limits $x \rightarrow \infty$ or $y \rightarrow \infty$ in Eq. 3.12. Moreover, when $t \in \mathbb{N}$, the $\operatorname{CDF}$ (3.12) coincides with Eq. 1.3 with $n=t$. Further, when $t \in(0,1]$, then $\left[t^{-}\right]=0$ and the representation (3.11) coincides with Eq. 3.3 with $\alpha=\langle t\rangle$ (as the min and the max over an empty set are $\infty$ and $-\infty$, respectively), with the CDF (3.12) turning into Eq. 3.4. Finally, for $t>1$, the indicator function in Eq. 3.12 takes on the value of 0, and the above CDF reduces to

$$
H(x, y)=\left\{\begin{array}{lr}
F^{n+\alpha}(y)-(F(y)-F(x))^{n}\left(F^{\alpha}(y)-1+\bar{F}^{\alpha}(x)\right) & \text { for } x<y, \\
F^{n+\alpha}(y) & \text { for } x \geq y,
\end{array}\right.
$$

where $n=\left[t^{-}\right] \in \mathbb{N}$ and $\alpha=\langle t\rangle \in(0,1]$. Note that the CDF (3.13) is actually well defined for any $n \in \mathbb{N}$ and any $\alpha \in[0,1]$, and reduces to the CDF of the extreme order statistics whenever $\alpha=0$ and $\alpha=1$ (corresponding to the sample size of $n$ and $n+1$, respectively).

The bivariate distributions discussed above can also be described through the copula representation (see Sklar 1959)

$$
H(x, y)=C\left(F_{X}(x), F_{Y}(y)\right), \quad x, y \in \mathbb{R},
$$

where $F_{X}(\cdot)$ and $F_{Y}(\cdot)$ are the marginal CDFs of $X$ and $Y$ in Eq. 3.1. If the underlying $\mathrm{CDF} F(\cdot)$ of the $\left\{X_{i}\right\}$ in Eq. 3.1 is a continuous function, then so are the marginal CDFs $F_{X}(\cdot)$ and $F_{Y}(\cdot)$ and the copula $C(\cdot, \cdot)$ is unique (see, e.g., Nelsen 1999, Theorem 2.3.3., p. 18). Straightforward calculations show that in this case the copula $C_{N}$ connected with the bivariate distribution (3.2) for $N$ with the PGF $G_{N}$ is given by

$$
C_{N}(u, v)= \begin{cases}u-G_{N}\left(G_{N}^{-1}(u)+G_{N}^{-1}(1-v)-1\right) & \text { for } G_{N}^{-1}(u)+G_{N}^{-1}(1-v)>1 \\ u & \text { for } G_{N}^{-1}(u)+G_{N}^{-1}(1-v) \leq 1\end{cases}
$$


In the Sibuya case, the copula $C_{\alpha}=C_{N_{\alpha}}$ connected with the bivariate distribution (3.3) is of the form

$$
C_{\alpha}(u, v)= \begin{cases}u-1+\left\{(1-u)^{1 / \alpha}+v^{1 / \alpha}\right\}^{\alpha} & \text { for }(1-u)^{1 / \alpha}+v^{1 / \alpha}<1, \\ u & \text { for }(1-u)^{1 / \alpha}+v^{1 / \alpha} \geq 1 .\end{cases}
$$

Note that although the copula function (3.14) is continuous on the unit square, the induced probability distribution may not be absolutely continuous. This has to do with the fact that the distribution of $(X, Y)$ may have a singular part on the diagonal, as it was discussed earlier. For example, in the Sibuya case (3.15), we have the following result in the spirit of Proposition 3.4, which can be derived by routine calculations.

Proposition 3.8 The copula function $C_{\alpha}$ in Eq. 3.15 admits the representation

$$
C_{\alpha}(u, v)=(1-\alpha) G_{\alpha}(u, v)+\alpha S_{\alpha}(u, v), \quad u, v \in[0,1],
$$

where

$$
G_{\alpha}(u, v)=\frac{1}{1-\alpha}\left\{\begin{array}{lr}
u-1+\left\{(1-u)^{1 / \alpha}+v^{1 / \alpha}\right\}^{\alpha}-\alpha v^{1 / \alpha} & \text { for }(1-u)^{1 / \alpha}+v^{1 / \alpha}<1, \\
u-\alpha\left(1-(1-u)^{1 / \alpha}\right) & \text { for }(1-u)^{1 / \alpha}+v^{1 / \alpha} \geq 1,
\end{array}\right.
$$

is the CDF of an absolutely continuous bivariate distribution on the unit square and

$$
S_{\alpha}(u, v)=\left(1-(1-u)^{1 / \alpha}\right) \wedge v^{1 / \alpha}, u, v \in[0,1],
$$

is the CDF of singular distribution on the unit square, describing the random vector $\left(1-(1-U)^{\alpha}, U^{\alpha}\right)$ with standard uniform $U$.

Three examples of the copulas (3.15) with $\alpha=0.25, \alpha=0.5$ (the discrete MittagLeffler case), and $\alpha=0.75$, along with their continuous and singular components, are presented in Fig. 1.

Remark 3.9 The above mixture representation is useful for generating random variates from the bivariate distribution on the unit square with the $\operatorname{CDF} C_{\alpha}$. Note that the singular part, given by the CDF $S_{\alpha}$, is supported on the curve $(1-u)^{1 / \alpha}+v^{1 / \alpha}=1$, $u, v \in[0,1]$, and its marginal distributions are both beta distributions (with parameters $1,1 / \alpha$ and $1 / \alpha, 1$, respectively). The PDF of the absolutely continuous part with the $\mathrm{CDF}$ (3.16) is given by

$g_{\alpha}(u, v)=\frac{1}{\alpha} \begin{cases}(1-u)^{\frac{1-\alpha}{\alpha}} v^{\frac{1-\alpha}{\alpha}}\left((1-u)^{\frac{1}{\alpha}}+v^{\frac{1}{\alpha}}\right)^{\alpha-2} & \text { for } 0<u<1,(1-u)^{1 / \alpha}+v^{1 / \alpha}<1, \\ 0 & \text { otherwise. }\end{cases}$

Further, the two marginal PDFs corresponding to Eq. 3.18 are

$g_{\alpha}^{(1)}(u)=\frac{1}{1-\alpha}\left(1-(1-u)^{\frac{1-\alpha}{\alpha}}\right), g_{\alpha}^{(2)}(v)=\frac{1}{1-\alpha}\left(1-v^{\frac{1-\alpha}{\alpha}}\right), \quad 0<u, v<1$,

in which we recognize the distributions of $1-W^{\frac{\alpha}{1-\alpha}}$ and $W^{\frac{\alpha}{1-\alpha}}$, respectively, where $W$ has beta distribution with the parameters $\frac{\alpha}{1-\alpha}$ and 2 . 

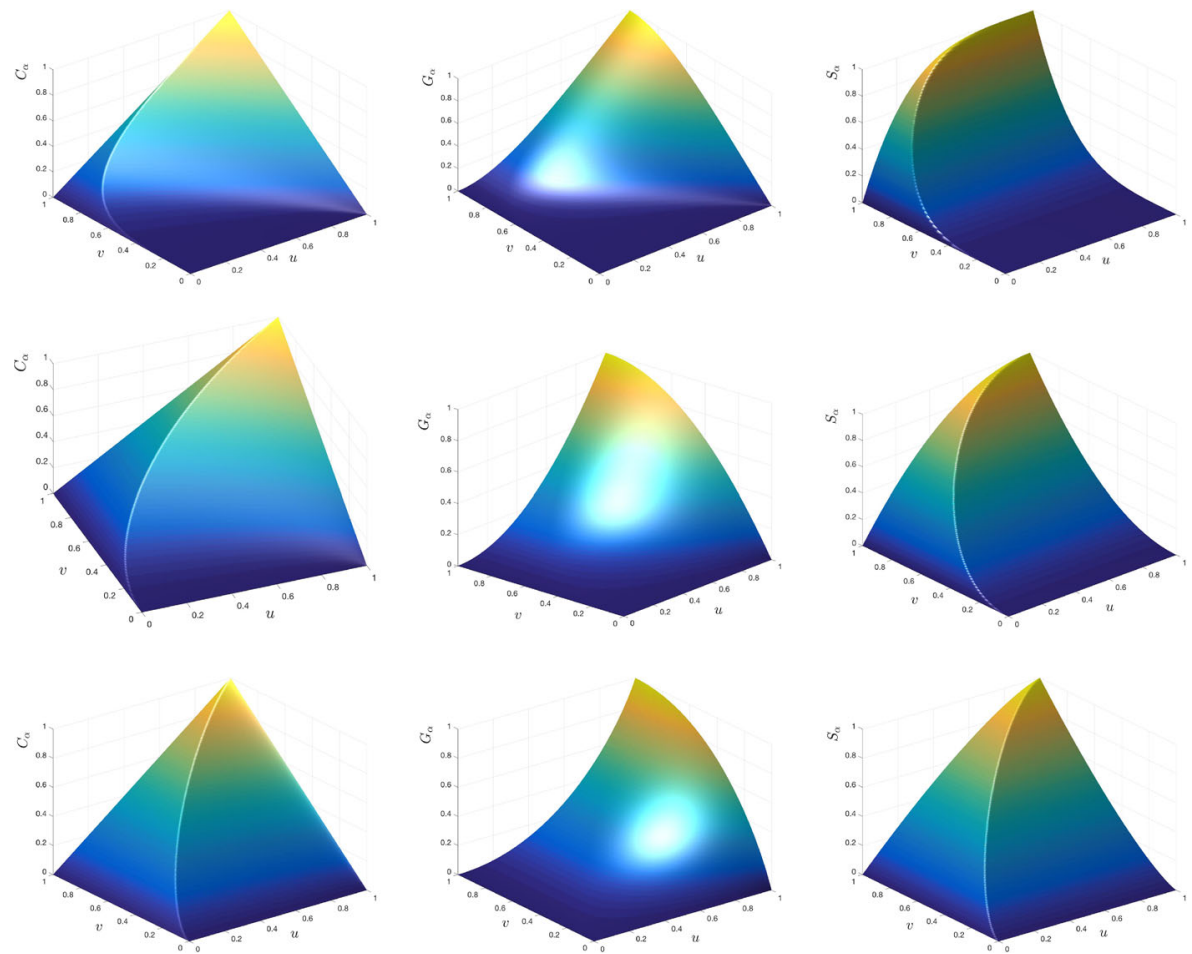

Fig. 1 Three cases of the copulas $C_{\alpha}$ (the first column), their continuous component $G_{\alpha}$ (the second column), and the singular component $S_{\alpha}$ (the third column): $\alpha=0.25-$ top, $\alpha=0.5-$ middle, and $\alpha=0.75-$ bottom

Finally, the two conditional CDFs corresponding to Eq. 3.18 are

$$
G_{\alpha}(v \mid u)=\frac{1-\frac{(1-u)^{\frac{1-\alpha}{\alpha}}}{\left((1-u)^{\frac{1}{\alpha}}+v^{\frac{1}{\alpha}}\right)^{1-\alpha}}}{1-(1-u)^{\frac{1-\alpha}{\alpha}}}, 0<u<1,0<v<\left(1-(1-u)^{\frac{1}{\alpha}}\right)^{\alpha}
$$

and

$$
G_{\alpha}(u \mid v)=\frac{\left((1-u)^{\frac{1}{\alpha}}+v^{\frac{1}{\alpha}}\right)^{\alpha-1}-1}{v^{\frac{\alpha-1}{\alpha}}-1}, 0<v<1,1-\left(1-v^{\frac{1}{\alpha}}\right)^{\alpha}<u<1 .
$$

The obtained results are utilized in an algorithm to simulate bivariate random variable $(U, V)$ from the copula $C_{\alpha}$. Three samples generated using the algorithm are presented in Fig. 2, while details on the algorithm are given in the Appendix. 

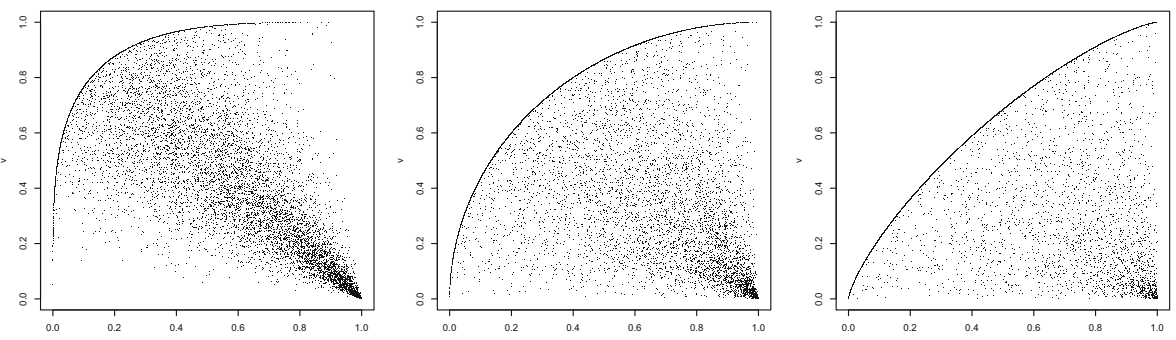

Fig. 2 Three samples of size $10^{4}$ from the copulas $C_{\alpha}$ with $\alpha=0.25$ (left panel), $\alpha=0.5$ (middle panel), and $\alpha=0.75$ (right panel)

In the more general case of Eq. 3.11 with $n=\left[t^{-}\right] \in \mathbb{N}$ and $\alpha=\langle t\rangle \in(0,1]$, the copula is

$C_{\alpha, n}(u, v)= \begin{cases}v & \text { for }(1-u)^{\frac{1}{\alpha+n}}+v^{\frac{1}{\alpha+n}} \leq 1, \\ v-\left\{(1-u)^{\frac{1}{\alpha+n}}+v^{\frac{1}{\alpha+n}}-1\right\}^{n}\left\{(1-u)^{\frac{\alpha}{\alpha+n}}+v^{\frac{\alpha}{\alpha+n}}-1\right\}\end{cases}$ otherwise.

This is a generalization of the min-max copula discussed in Schmitz (2004) (see also Avérous et al. 2005 for more general results), to which it reduces for boundary values $\alpha=0,1$. Four examples of such copulas are shown in Fig. 3. We note that in this case $(n \in \mathbb{N})$ they do not have singular components.

\section{Further examples and a duality of distributions on $\mathbb{N}$}

A variety of new families of distributions can be generated from a "base" CDF $F$ (or SF $\bar{F}$ ) and an $\mathbb{N}$-valued random variable $N$ with PGF $G_{N}$ viz. Lemma 2.1. One wellknown example of such a construction is the Marshall-Olkin scheme introduced in Marshall and Olkin (1997), where a SF $\bar{F}$ generates a family of SFs given by

$$
S(x ; \alpha)=\frac{\alpha \bar{F}(x)}{1-(1-\alpha) \bar{F}(x)}, \quad x \in \mathbb{R}, \quad \alpha \in \mathbb{R}_{+} .
$$

For $\alpha=p \in(0,1)$, the above SF is of the form $G_{N}(\bar{F}(x))$, where $G_{N}$ is the PGF of a geometric random variable $N$ with parameter $p$, given by the PDF

$$
\mathbb{P}(N=n)=p(1-p)^{n-1}, \quad n \in \mathbb{N} .
$$

A straightforward calculation shows that, when $\alpha>1$, then the CDF corresponding to Eq. 4.1 is of the form $G_{N}(F(x))$, where $F(x)=1-\bar{F}(x)$ and $G_{N}$ is the PGF of a geometric random variable with parameter $p=1 / \alpha$. Thus, in view of Lemma 2.1, the family of distributions defined viz. Eq. 4.1 is generated by geometric minima $(0<\alpha<1)$ and geometric maxima $(\alpha>1)$ of IID random variables with the base SF $\bar{F}$, as noted in Marshall and Olkin (1997).

Another example of this construction is connected with the so-called transmuted distributions, which have populated the literature since the introduction of the 

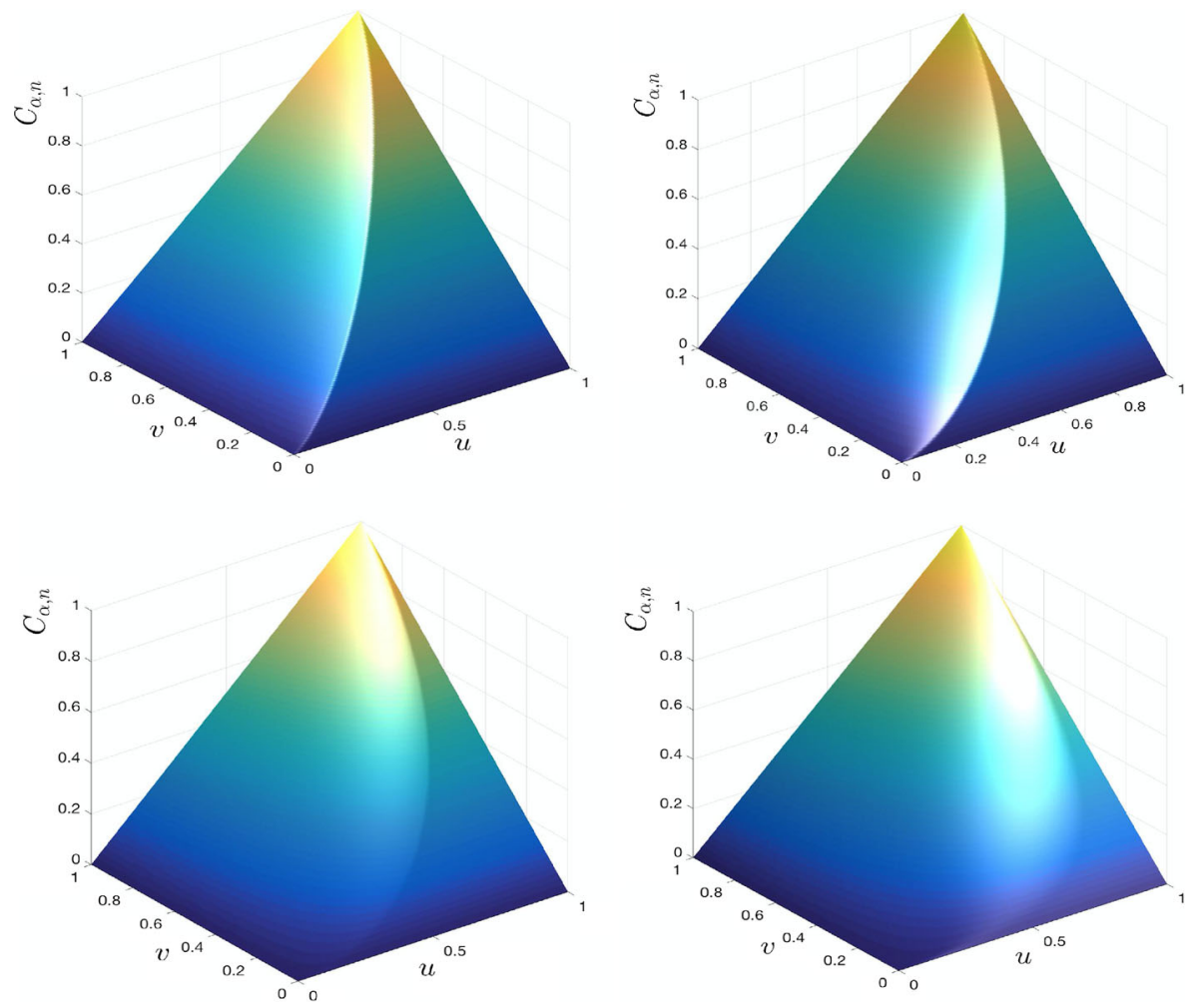

Fig. 3 Four cases of the copulas $C_{\alpha, n}: \alpha=0.25, n=1$ - top-left, $\alpha=0.5, n=1$ - top-right, $\alpha=0.75$, $n=1$ - bottom-left, $\alpha=0.5, n=2$ - bottom-right

quadratic rank transmutation map in Shaw and Buckley (2009). The latter, defined via

$$
u \rightarrow u+\alpha u(1-u), \quad u \in[0,1], \alpha \in[-1,1],
$$

is used to transform a base $\mathrm{CDF} F$ into its transmuted version $F_{\alpha}$, where

$$
F_{\alpha}(x)=(1+\alpha) F(x)-\alpha F^{2}(x), \quad x \in \mathbb{R} .
$$

As recently shown in Kozubowski and Podgórski (2016), the CDF in Eq. 4.4 is of the form $G_{-\alpha}(F(x))$ for $\alpha \in[-1,0]$ and of the form $1-G_{\alpha}(1-F(x))$ for $\alpha \in[0,1]$, where

$$
G_{p}(s)=s(1-p+p s), \quad s \in[0,1],
$$

is the PGF of a Bernoulli distribution with parameter $p \in[0,1]$ shifted up by one. Again, by Lemma 2.1, the family of distributions defined viz. Eq. 4.4 is generated by random minima $(0 \leq \alpha \leq 1)$ and random maxima $(-1 \leq \alpha \leq 0)$ of IID random variables with the base CDF $F$. See Kozubowski and Podgórski (2016) for more information and extensive references.

The above examples illustrate the fact that virtually any $\mathbb{N}$-valued random variable $N$ generates a new probability distribution from that of a base random variable 
$X$ connected with the maximum (or minimum) of $N$ IID copies of $X$. In view of this, we pose the following question: If $X$ is coupled with $N$ as in Lemma 2.1 to generate a new distribution of $Y \stackrel{d}{=} \max \left\{X_{1}, \ldots, X_{N}\right\}$, then can this process be reversed, so that for some $\mathbb{N}$-valued random variable $\tilde{N}$ we would also have $X \stackrel{d}{=} \min \left\{Y_{1}, \ldots, Y_{\tilde{N}}\right\}$, where the $\left\{Y_{i}\right\}$ are IID copies of $Y$, independent of $\tilde{N}$ ? Similarly, if the new distribution is generated via a random minimum rather than maximum, so that, $Y \stackrel{d}{=} \min \left\{X_{1}, \ldots, X_{N}\right\}$, then is there an integer-valued random variable $\tilde{N}$ so that we would have $X \stackrel{d}{=} \max \left\{Y_{1}, \ldots, Y_{\tilde{N}}\right\}$ ? In view of our results in Section 2, it is not hard to see that in order for these conditions to hold the PGFs of $N$ and $\tilde{N}$ need to satisfy the relation

$$
G_{\tilde{N}}\left(1-G_{N}(s)\right)=1-s, \quad s \in[0,1]
$$

so that

$$
G_{\tilde{N}}(s)=1-G_{N}^{-1}(1-s), \quad s \in[0,1] .
$$

In turn, if the quantity on the right-hand-side of Eq. 4.7 is a genuine PGF, then the process of taking random maxima (or minima) can be reversed as described above. This motivates the following definition.

Definition 4.1 If a probability distribution on $\mathbb{N}$ with the PGF $G_{N}$ yields through Eq. 4.7 a genuine PGF, then such a pair of distributions on $\mathbb{N}$ is said to be in the duality relation with respect to the operation of random $\mathrm{min} / \mathrm{max}$.

While the random $\mathrm{min} / \mathrm{max}$ duality is in fact the property of distributions residing on $\mathbb{N}$ we carry over this terminology to random variables with values in $\mathbb{N}$ for which the distributions are in the introduced duality relation. Note that the notion of being a dual is symmetric, that is if $\tilde{N}$ is a dual to $N$ then in turn $N$ is a dual of $\tilde{N}$. Further, as noted above, the four operations of taking random $\min / \max$ with either $N$ or $\tilde{N}$ are reversible and recover the original distribution, as stated in the following result.

Proposition 4.2 If $N$ and $\tilde{N}$ are a dual pair of $\mathbb{N}$-valued random variables, then for any double-sequence $\left\{X_{i j}\right\}$ of IID random variables we have

$$
X_{11} \stackrel{d}{=} \bigwedge_{i=1}^{N} \bigvee_{j=1}^{\tilde{N}_{i}} X_{i j} \stackrel{d}{=} \bigvee_{i=1}^{N} \bigwedge_{j=1}^{\tilde{N}_{i}} X_{i j} \stackrel{d}{=} \bigwedge_{i=1}^{\tilde{N}} \bigvee_{j=1}^{N_{i}} X_{i j} \stackrel{d}{=} \bigvee_{i=1}^{\tilde{N}} \bigwedge_{j=1}^{N_{i}} X_{i j}
$$

with the $\left\{X_{i j}\right\}$ being independent of all the $\mathbb{N}$-valued random variables that appear in Eq. 4.8.

Let us now present several dual pairs related to Sibuya and other standard probability distributions. 


\subsection{Deterministic/Sibuya pair}

If the random variable $N$ is a deterministic one, taking on the value of $n \in \mathbb{N}$ with probability 1, then the PGF of $N$ is $G_{N}(s)=s^{n}$ while the dual PGF in Eq. 4.7 becomes

$$
G_{\tilde{N}}(s)=1-(1-s)^{1 / n}, \quad s \in[0,1],
$$

which we recognize to be Sibuya distributed with parameter $1 / n \in(0,1]$. In turn, the dual to a Sibuya variable with parameter $1 / n$ is a deterministic variable equal to $n$. However, if $N$ is taken to be Sibuya with parameter $\alpha$ where $1 / \alpha$ is not an integer, then the quantity $G_{\tilde{N}}(s)=s^{1 / \alpha}$ is not a valid PGF. Thus, a Sibuya random variable has no dual unless $1 / \alpha$ is an integer.

\subsection{Shifted Bernoulli/tilted Sibuya pair}

Let $N$ have a Bernoulli distribution with parameter $p \in[0,1]$ shifted up by one, given by the PGF (4.5). Then routine albeit lengthy calculations lead to the dual PGF,

$$
G_{\tilde{N}}(s)=\frac{1+p}{2 p}\left\{1-\left(1-\frac{4 p}{(1+p)^{2}} s\right)^{\frac{1}{2}}\right\}, s \in[0,1],
$$

which, upon expending the square root above into a power series, produces the dual PDF

$$
\mathbb{P}(\tilde{N}=n)=\frac{1+p}{2 p}\left(\frac{4 p}{(1+p)^{2}}\right)^{n}\left(\begin{array}{c}
1 / 2 \\
n
\end{array}\right)(-1)^{n+1}, n \in \mathbb{N} .
$$

This can be seen as an exponentially tilted Sibuya distribution of $N_{\alpha}$ given by Eq. 2.3 with $\alpha=1 / 2$,

$$
\mathbb{P}(\tilde{N}=n)=\frac{r^{n} \mathbb{P}\left(N_{\alpha}=n\right)}{\mathbb{E} r^{N_{\alpha}}}, n \in \mathbb{N},
$$

where $r=4 p /(1+p)^{2}$.

\subsection{Poisson/logarithmic pair}

Consider a Poisson random variable with parameter $\lambda$ truncated below at 1 , given by the PDF

$$
\mathbb{P}(N=n)=\frac{e^{-\lambda} \lambda^{n}}{n !\left(1-e^{-\lambda}\right)}, \quad n \in \mathbb{N},
$$

and the PGF

$$
G_{N}(s)=\frac{e^{-\lambda(1-s)}-e^{-\lambda}}{1-e^{-\lambda}}, \quad s \in[0,1] .
$$

Straightforward algebra shows that the dual PGF in Eq. 4.7 becomes

$$
G_{\tilde{N}}(s)=-\frac{1}{\lambda} \log \left\{1-\left(1-e^{-\lambda}\right) s\right\}, \quad s \in[0,1],
$$


which, upon expending the logarithm above into a power series, is seen to describe a logarithmic distribution with the PDF

$$
\mathbb{P}(\tilde{N}=n)=-\frac{(1-q)^{n}}{n \log q}, n \in \mathbb{N}, \quad q=e^{-\lambda} \in(0,1) .
$$

\subsection{Negative binomial/negative binomial pair}

Consider a negative binomial (NB) distribution with parameters $r \in \mathbb{R}_{+}$and $p \in$ $(0,1]$, given by the PDF

$$
p_{n}=\left(\begin{array}{c}
r+n-1 \\
r
\end{array}\right) p^{r}(1-p)^{n}, \quad n \in \mathbb{N}_{0}=\{0,1, \ldots\} .
$$

Let $N$ have the above distribution truncated below at 1 , with the probabilities given by $p_{n} /\left(1-p^{r}\right), n \in \mathbb{N}$, and the PGF

$$
G_{N}(s)=\frac{1}{1-p^{r}}\left\{\left(\frac{p}{1-(1-p) s}\right)^{r}-p^{r}\right\}, \quad s \in[0,1] .
$$

After routine calculation, we find the dual PGF to be

$$
G_{\tilde{N}}(s)=\frac{1}{1-p}\left\{\left(\frac{p^{r}}{1-\left(1-p^{r}\right) s}\right)^{\frac{1}{r}}-p\right\}, \quad s \in[0,1],
$$

which we recognize to be a NB distribution with parameters $1 / r$ and $p^{r}$, truncated below at 1 . Thus, NB distributions with parameters $r, p$ and $1 / r, p^{r}$, both truncated below at 1 , form a dual pair in our scheme. In particular, when $r=1$, both distributions of the dual pair are the same geometric distributions, given by the PDF (4.2). This has an interesting interpretation: If $N$ is geometrically distributed and $Y$ is obtained via a maximum (minimum) of $N$ IID variables $\left\{X_{i}\right\}$ and in turn we take the minimum (maximum) of $N$ IID copies of $Y$, then we recover the distribution of the $\left\{X_{i}\right\}$.

\section{The max-min process on the unit interval}

The Sibuya distribution with parameter $t=\alpha, t \in[0,1]$, can be obtained as the marginal distribution of a Sibuya random process on $[0,1]$,

$$
N(t)=\min \left\{n \in \mathbb{N}: U_{n} \leq t / n\right\}, \quad t \in[0,1],
$$

which was introduced in Kozubowski and Podgórski (2017). Here, the $\left\{U_{n}\right\}$ are IID standard uniform random variables and $N(0)=\infty$. Clearly, since $\mathbb{P}\left(n U_{n} \leq t\right)=t / n$ for each $t \in(0,1]$, the variable $N(t)$ has the Sibuya distribution (2.3) with $\alpha=t$.

As shown in Kozubowski and Podgórski (2017), $N(t)$ is a right-continuous, purejump, and non-increasing process, with an infinite number of jumps in any (right) neighborhood of zero. In addition, the location of the jumps and their sizes are closely related to the magnitudes and the locations of the records connected with the $\left\{n U_{n}\right\}$, where the value that is smaller than all the previous values sets a new record. These are described via the pairs $R_{k}$ and $T_{k}$, which are the integer index in the sequence 
$\left\{n U_{n}\right\}$ at which the $k$ th record occurs and the size (the value among the $\left\{n U_{n}\right\}$ ) of this record, respectively. By assumption, the first value is a record, so that $R_{1}=1$ and $T_{1}=U_{1}$. Further, we let $G_{k+1}=R_{k+1}-R_{k}$ be the inter-arrival times between successive records (measured in the positive integer values). With this notation, the Sibuya process (5.1) admits the following representation for $t \in[0,1]$ :

$$
\begin{aligned}
N(t) & =1+\sum_{k=1}^{\infty} G_{k+1} \mathbb{I}_{(t, 1]}\left(T_{k}\right) \\
& =\sum_{k=1}^{\infty} R_{k} \mathbb{I}_{\left(T_{k}, T_{k-1}\right]}(t),
\end{aligned}
$$

where $\mathbb{I}_{A}(\cdot)$ is the indicator function of a set $A$ and we set $T_{0}=1$. Viewing from right to left, the random process $N(t)$ starts with $N(1)=1$ and then jumps up by $G_{k+1}$ at every record value $T_{k}$. Other than that, the values of $N(t)$ are constant on the intervals $\left[T_{k}, T_{k-1}\right)$, and $N\left(T_{k}\right)=R_{k}$. This representation of the Sibuya process can be more explicitly expressed using the generalized Sibuya distribution.

Following Kozubowski and Podgórski (2017), recall that a variable is generalized Sibuya distributed with parameters $\alpha$ and $v, 0<\alpha<v+1$, denoted by $G_{1}(\alpha, v)$, if it is the waiting time for the first success in a series of Bernoulli trials with the probability of a success in the $n$th trial being $\alpha /(v+n)$. The PGF function of such a random variable is given by

$$
G_{\alpha, v}(s)=\frac{\alpha}{v+1} s(1-s)^{\alpha} F(v+1, \alpha+1 ; v+2 ; s), \quad 0<s<1,
$$

where

$$
F(a, b ; c ; t)=\sum_{n=0}^{\infty} \frac{(a)_{n}(b)_{n}}{(c)_{n}} \frac{t^{n}}{n !},|t|<1,
$$

is the Gauss hypergeometric function and

$$
(x)_{n}= \begin{cases}x(x+1) \cdots(x+n-1) & \text { for } n \geq 1, \\ 1 & \text { for } n=0\end{cases}
$$

is the (rising) Pochhammer symbol.

Then it follows from Proposition 15 of Kozubowski and Podgórski (2017) that the times of jumps and their cumulated sizes are represented by a homogenous Markov process $\left(R_{k}, T_{k}\right), k \in \mathbb{N}$, defined through

$$
\begin{gathered}
\left(R_{1}, T_{1}\right) \stackrel{d}{=}\left(1, U_{1}\right), \\
\left.\left(R_{k}, T_{k}\right)\right|_{\left(R_{k-1}, T_{k-1}\right)=(r, t)} \stackrel{d}{=}\left(r+S_{k}, t U_{k}\right), \quad k>1,
\end{gathered}
$$

where the $\left\{U_{k}\right\}, k \in \mathbb{N}$, are IID standard uniform variables, while $S_{k}$ has generalized Sibuya distribution $G S_{1}(t, r)$ and is independent of $U_{k}$. As before, the notation " $=$ " stands for equality in distribution. Twenty samples of the Sibuya process are presented in Fig. 4. More information about this process can be found in Kozubowski and Podgórski (2017).

The above Sibuya process can be used to construct a bivariate process on $[0,1]$ via Corollary 3.2. The process will be naturally referred to as the max-min process, 


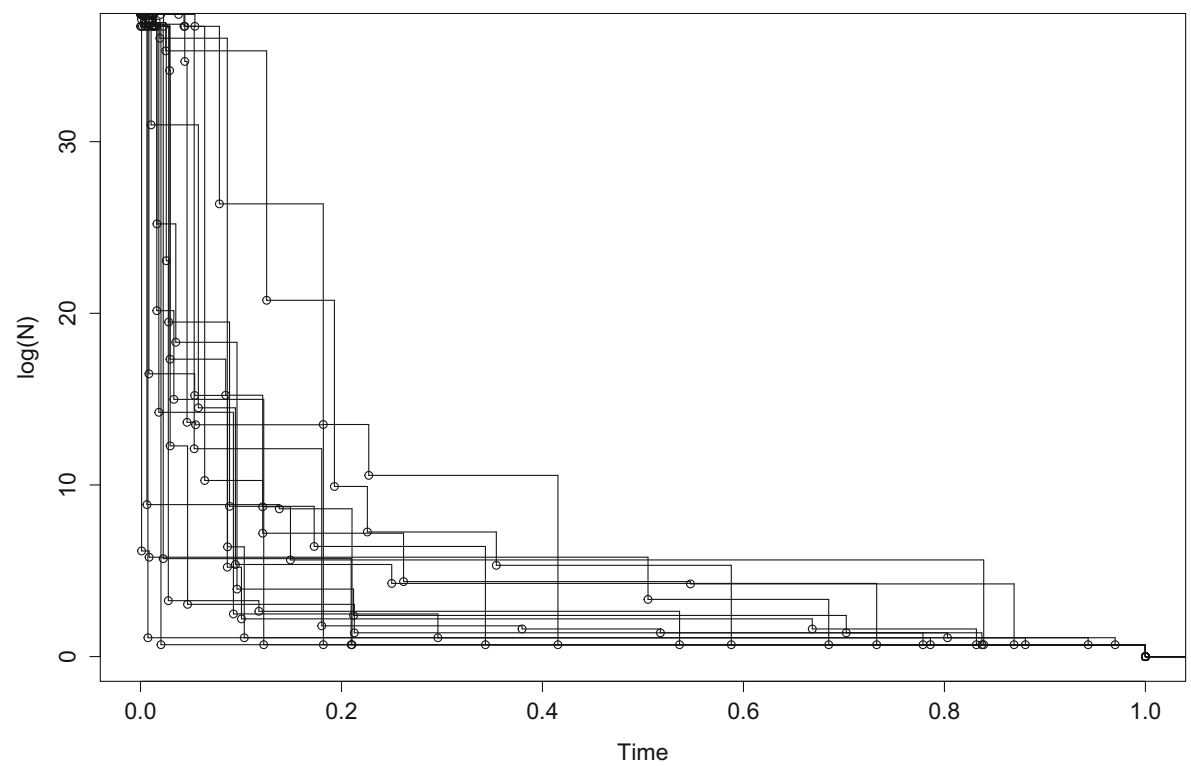

Fig. 4 Twenty samples of the Sibuya process on the logarithmic scale in values. The abscissae of the circles indicate locations of jumps and their ordinates are the logarithmic values of the Sibuya process after the jump

and it will be first defined on the unit interval $[0,1]$. Starting with a sequence of IID random variables $\left\{X_{n}\right\}$ with the common $\operatorname{CDF} F$ and $\operatorname{SF} \bar{F}$, we let

$$
(X(t), Y(t))=\left(\bigvee_{j=1}^{N(t)} X_{j}, \bigwedge_{j=1}^{N(t)} X_{j}\right), t \in[0,1]
$$

where $N(t)$ is the Sibuya process, independent of $\left\{X_{n}\right\}$. It follows that for each $t \in$ $(0,1)$ the marginal distributions of $(X(t), Y(t))$ are given by Eq. 3.4 with $\alpha=t$. In particular, the marginal SF of $X(t)$ is $[\bar{F}(\cdot)]^{t}$, while the marginal CDF of $Y(t)$ is $[F(\cdot)]^{t}$. Next, we provide an insight into the full set of finite dimensional distributions through a convenient Markovian representation of the max-min process. We start with a representation of the max-min process through a four-dimensional hidden Markov process of jumps and their locations.

Theorem 5.1 Let $\mathbf{J}_{n}=\left(M_{n}, m_{n}, R_{n}, T_{n}\right), n \in \mathbb{N}$, be a homogeneous Markov process defined through

$$
\begin{gathered}
\mathbf{J}_{1} \stackrel{d}{=}\left(X_{1}, X_{1}, 1, U_{1}\right), \\
\left.\mathbf{J}_{k}\right|_{\mathbf{J}_{k-1}=(M, m, r, t)} \stackrel{d}{=}\left(M \vee W_{k}, m \wedge w_{k}, r+S_{k}, t U_{k}\right), \quad k>1,
\end{gathered}
$$


where $\left(W_{k}, w_{k}\right), S_{k}$, and $U_{k}$ are mutually independent and

$$
\begin{aligned}
\mathbb{P}\left(W_{k} \leq x, w_{k} \leq y\right) & =\left\{\begin{array}{l}
G_{t, r}(F(x))-G_{t, r}(F(x)-F(y)) \text { for } x>y, \\
G_{t, r}(F(x))
\end{array}\right. \\
S_{k} & \sim G_{1}(t, r),
\end{aligned}
$$

with $G_{t, r}$ being the PGF of generalized Sibuya distribution given in Eq. 5.3. Then the sequences $\left\{M_{k}\right\}$ and $\left\{m_{k}\right\}$ are non-decreasing and non-increasing, respectively, and the max-min process (5.5) can be represented as follows:

$$
(X(t), Y(t)) \stackrel{d}{=} \sum_{k=1}^{\infty}\left(M_{k}, m_{k}\right) \mathbb{I}_{\left(T_{k}, T_{k-1}\right]}(t) .
$$

Remark 5.2 We have referred to the above representation as a hidden Markov one since the values of $S_{n}$ are not observed in trajectories of the min-max process, and the three sequences $\left\{M_{n}\right\},\left\{m_{n}\right\}$, and $\left\{T_{n}\right\}$ alone, which are explicitly used in the representation, do not have Markovian structure.

As it was remarked in the introduction, there are some similarities between $Y(t)$ and the maximal process $M(t)$ given by Eq. 1.5. Embedding the records in the record process has some resemblance to embedding the minima $S_{n}$ or the maxima $M_{n}$ (given by Eqs. 1.1 and 1.2, respectively), into the extremal processes, as described in Resnick (1987). Since the extremal process has been extensively studied and constitutes one of the fundamental concepts in the extremal value theory, it is important to explain the relation between the max-min process and the extremal process. Probably, the most direct way of comparing the two is through the structure of the jumps and their locations.

Let us consider the following two dimensional process

$$
\begin{aligned}
& \left(J_{1}, T_{1}\right) \stackrel{d}{=}\left(F^{-1}\left(V_{1}\right), U_{1}\right), \\
& \left(J_{k}, T_{k}\right) \stackrel{d}{=}\left(F^{-1}\left(V_{k}^{\frac{1}{U_{1} \ldots U_{k-1}}} \ldots V_{2}^{\frac{1}{U_{1}}} V_{1}\right), U_{k} \ldots U_{2} U_{1}\right), \quad k>1,
\end{aligned}
$$

where the $\left\{V_{n}\right\}$ and the $\left\{U_{n}\right\}$ are independent standard uniform random variables while $F^{-1}$ is the generalized inverse of the CDF $F$. It is not hard to argue (see Dwass 1966; Resnick and Rubinovitch 1973) that the sequence $\left\{J_{k}\right\}$ is decreasing and the extremal process admits the representation

$$
M(t) \stackrel{d}{=} \sum_{k=1}^{\infty} J_{k} \mathbb{I}_{\left(T_{k}, T_{k-1}\right]}(t),
$$

where, by definition, $T_{0}=1$.

One can now compare the above representation with the one for $Y(t)$ given in Theorem 5.1. First, we note that the values of both processes are the same until the first jump $T_{1}$ of $M(t)$ (assuming that $X_{1}=F^{-1}\left(V_{1}\right)$ ). Moreover, both processes are nondecreasing with each having an infinite number of jumps, but a finite number of them 
outside any neighborhood of zero. We observe that every location of a jump in $Y(t)$ is also a location of a jump in $M(t)$ (if the same $\left\{U_{k}\right\}$ are used for both the processes). However, the opposite is not true, since not every $T_{k}$ is a location of a jump for $Y(t)$. The jump at the $k$ th location $T_{k}$ (counted from the right to the left) would not occur when the value of $w_{k}$ given in Eq. 5.7 is smaller than the value $M\left(T_{k-1}\right)-$ an event that has non-zero probability at each $T_{k}$. Thus, while the $\left\{J_{k}\right\}$ are strictly decreasing, the $\left\{m_{k}\right\}$ are only non-increasing. Additionally, note that such a relatively simple Markovian structure of $Y(t)$ is not available for the extremal process. We conclude that the two processes are fundamentally different.

The jump values $\left(M_{n}, m_{n}\right)$ are interesting on their own and we explore them in the next result.

Proposition 5.3 The random vector $\left(W_{k}, w_{k}\right), k \in \mathbb{N}$, in the Markovian process (5.7) has a distributional representation

$$
\left(W_{k}, w_{k}\right) \stackrel{d}{=}\left(F^{-1}\left(\frac{E_{1}+G_{k}}{E_{1}+E_{2}+G_{k}}\right), F^{-1}\left(\frac{E_{1}}{E_{1}+E_{2}+G_{k}}\right)\right),
$$

where random variables $E_{1}, E_{2}$, and $G_{k}$ are mutually independent. Moreover, $E_{1}$ and $E_{2}$ are standard exponential while $G_{k}=P_{1}+\cdots+P_{S_{k}-1}$, where the $\left\{P_{i}\right\}$ are IID standard exponential variables.

Remark 5.4 The above result, coupled with Theorem 5.1, can be utilized to simulate the max-min process. Due to the Markovian structure, it is enough to address simulation of $S_{k}$ and $G_{k}$. Generally, as $k$ increases, one will observe very quickly extremely large values of $S_{k}$ - a variable that for the range of parameters involved does not have the mean. This can be used to replace $G_{k}$ by $S_{k}$ due to the fact that $P_{1}+\cdots+P_{N} \approx N$ for large $N$. Thus, simulation can be substantially reduced to simulation of the generalized Sibuya variables $S_{k}$. The simulation of $S_{k}$, while non-trivial, was discussed in Kozubowski and Podgórski (2017).

As we stated above, the jumps of the process $N(t)$ are extremely large. In our simulations, they were on the order of $10^{10}$ or higher for $t<0.05$. Thus, based on classical extreme value theory, one might expect that the extremal process given in Eq. 5.5 would exhibit some asymptotical distributional invariance. Namely, by extremal types theorem, under certain conditions on the $\left\{X_{j}\right\}$ and when appropriately normalized, the maximum $M_{n}=\bigvee_{j=1}^{n} X_{j}$ converges in distribution to one of the three standard extreme value distributions. For example, if the $\left\{X_{j}\right\}$ were standard Pareto distributed with the SF $\bar{F}(x)=1 \wedge x^{-\alpha}$, then the limiting distribution of $M_{n} / n^{1 / \alpha}$ would be the Fréchet distribution given by the $\operatorname{CDF} \exp \left(-x^{-\alpha}\right), x>0$. However despite the fact that $N(t)$ is very large when $t$ is close to zero, the process $X(t)=\bigvee_{j=1}^{N(t)} X_{j}$ does not lose the effect of the entire SF of $X$ on its SF, which is $\bar{F}^{t}$. In our special case, this is still Pareto distribution, but with the tail parameter $\alpha t$, and thus with much heavier power tail than that of the Fréchet distribution. 
The above considerations lead to a question about the asymptotics of $X(t)$ at zero. Namely, are there any normalizing functions $a(t)$ and $b(t)$ such

$$
Z(t)=(X(t)-b(t)) / a(t)
$$

has a non-degenerate limiting distribution as $t$ approaches zero? This is normally the case when the random index $N(t)$ tends to infinity while a scaled version of it, $c(t) N(t)$, has a non-zero limiting distribution (see, e.g., Embrechts et al. 1997, Section 4.3). However, as shown below, the Sibuya distribution $N(t)$ increases so fast as $t$ gets close to zero, that no normalization $c(t)$ can make it converge to a non-zero limit.

Proposition 5.5 For $p \in(0,1)$, let $N_{p}$ have a Sibuya distribution (2.3) with parameter $\alpha=p$, and suppose that $c_{p} N_{p} \stackrel{d}{\rightarrow} Z$ as $p \rightarrow 0$ for some $c_{p}>0$. Then $\mathbb{P}(Z=0)=1$.

In view of the above result, one may suspect that the quantity $Z(t)$ in Eq. 5.11 can never converge to an extreme value (or other non-degenerate) distribution as $t$ approaches zero. As shown below, this is indeed the case. To see this, consider again standard Pareto distributed $\left\{X_{i}\right\}$ and assume to the contrary that there exist functions $a(t)$ and $b(t)$ such that the distribution of $Z(t)$ has a non-degenerate limit at zero. If this was so, then $a(t)$ would have to converge to infinity, as otherwise $b(t)$ would have to converge to infinity to ensure that the $\mathrm{SF} 1 \wedge(x a(t)+b(t))^{-\alpha t}$ would converge to a SF. However such a limit would be independent of $x$, and thus it would not constitute a proper distribution. Further, note that $b(t) / a(t)$ must converge to infinity as well, since otherwise $1 \wedge(x a(t)+b(t))^{-\alpha t}=1 \wedge x^{-\alpha t} a(t)^{-\alpha t}\left(1+\frac{b(t)}{x a(t)}\right)^{-\alpha t}$ would converge to a constant independent of $x$. However, if both $a(t)$ and $b(t) / a(t)$ converge to infinity, then $1 \wedge x^{-\alpha t} b(t)^{-\alpha t}\left(\frac{1}{x}+\frac{a(t)}{b(t)}\right)^{-\alpha t}$ will have a limit not dependent on $x$ and thus not a proper distribution function. We conclude that no extremal-type theorem holds for $X(t)=\bigvee_{j=1}^{N(t)} X_{j}$ as $t$ converges to zero.

\section{The max-min process on the positive half-line}

The rest of the paper is devoted to definition and discussion of the max-min process extended from the unit interval to the positive half-line. The main tool for the extension is Proposition 3.6, but to motivate the approach we start with a discussion of the extremal process. As in Section 2, a real $t$ is split into its integer part $\left[t^{-}\right]$and its fractional part $\langle t\rangle=t-\left[t^{-}\right]$, defined in Eq. 2.6. It is not difficult to argue that the extremal process given in Eq. 1.5 can then be written as

$$
M(t) \stackrel{d}{=} \bigvee_{j=1}^{\left[t^{-}\right]} X_{j} \vee \max \left\{H_{j}^{\left(\left[t^{-}\right]\right)} ; \tau_{j}^{\left(\left[t^{-}\right]\right)} \leq\langle t\rangle\right\}
$$


where $\left(H_{i}^{(j)}, \tau_{i}^{(j)}\right)_{i \in \mathbb{N}}, j \in \mathbb{N}_{0}$, are independent copies of the jump sizes $\left\{H_{i}\right\}$ and the jump locations $\left\{\tau_{i}\right\}$ in the unit interval $[0,1]$, while $X_{j}=\max \left\{H_{i}^{(j-1)}, i \in \mathbb{N}\right\}$. Since these copies correspond to independent copies of a Lévy process, characterized by the jump sizes/location distributions, we can associate to each such a copy an independent extremal process, say $M_{j}(t)$, on the unit interval. Thus, it is easy to argue that the extremal process can be also written as

$$
\begin{aligned}
M(t) & \stackrel{d}{=} \bigvee_{j=1}^{\left[t^{-}\right]} X_{j} \vee M_{\left[t^{-}\right]}(\langle t\rangle) \\
& \stackrel{d}{=} \sum_{k=1}^{\infty}\left(\bigvee_{j=1}^{\left[t^{-}\right]} X_{j} \vee J_{k}^{\left(\left[t^{-}\right]\right)}\right) \mathbb{I}_{\left(T_{k}^{\left(\left[t^{-}\right]\right)}, T_{k-1}^{\left(\left[t^{-}\right]\right)}\right]}(\langle t\rangle),
\end{aligned}
$$

where, for given $j$, the process $M_{j}$ is independent of $X_{r}=F^{-1}\left(V_{1}^{(r-1)}\right), r \leq j$, while the sequences of pairs $\left(J_{k}^{(j)}, T_{k}^{(j)}\right)_{k \in \mathbb{N}}, j \in \mathbb{N}_{0}$, correspond to the representations of the extremal process copies as given in Eq. 5.9. Now, motivated by this structure of the extremal process, one could attempt to mimic it to extend the second coordinate of the max-min process by writing

$$
\begin{aligned}
Y(t) & \stackrel{d}{=} \bigvee_{j=1}^{\left[t^{-}\right]} X_{j} \vee Y_{\left[t^{-}\right]}(\langle t\rangle), \\
& \stackrel{d}{=} \sum_{k=1}^{\infty}\left(\bigvee_{j=1}^{\left[t^{-}\right]} X_{j} \vee m_{k}^{\left(\left[t^{-}\right]\right)}\right) \mathbb{I}_{\left(T_{k}^{\left(\left[t^{-}\right]\right)}, T_{k-1}^{\left(\left[t^{-}\right]\right)}\right]}(\langle t\rangle),
\end{aligned}
$$

where $X_{j}=X_{1}^{(j-1)}$, the $\left\{Y_{j}\right\}$ are independent copies of the second coordinate of the max-min process defined on the unit interval, and $\left(m_{k}^{(j)}, T_{k}^{(j)}\right)_{k \in \mathbb{N}}$ are the corresponding jump sizes and their locations as given in the representation (6.5). The extension of the max-min process that is given below yields the above analog. However, it is obtained without any direct reference to the extremal process. Instead, we use the properties of the maxima and minima with a Sibuya number of terms.

We start with a generic construction of a bivariate process that has marginal distributions defined by $\bar{F}^{t}$ and $F^{t}$. Consider a sequence $\left\{N^{(i)}(t)\right\}, i \in \mathbb{N}$, of independent Sibuya processes, which are independent of a sequence $\left\{X_{i}\right\}$ of IID random variables with the CDF $F$.

In addition, assume that, for each $n$, there is a sequence of IID random variables $X_{i}^{(n)}, i \in \mathbb{N}$, that is independent of $X_{1}, \ldots, X_{n}$ as well as of $N^{(n)}$. We set

$$
(X(t), Y(t))=\left(\bigwedge_{j=1}^{\left[t^{-}\right]} X_{j} \wedge \bigvee_{i=1}^{{ }^{\left(\left[t^{-}\right]\right)}(\langle t\rangle)} X_{i}^{\left(\left[t^{-}\right]\right)}, \bigvee_{j=1}^{\left[t^{-}\right]} X_{j} \vee \bigwedge_{i=1}^{{ }^{\left(\left[t^{-}\right]\right)}(\langle t\rangle)} X_{i}^{\left(\left[t^{-}\right]\right)}\right), t \in(0, \infty)
$$


For $t \in(0,1)$, where we have $\left[t^{-}\right]=0$ and $\langle t\rangle=t$, the minimum $\bigwedge_{j=1}^{0} X_{j}$ and the maximum $\bigvee_{j=1}^{\left[t^{-}\right]} X_{j}$ above are understood as $\infty$ and $-\infty$, respectively.

The following result summarizes fundamental properties of a process having the structure (6.4).

Theorem 6.1 Let $(X(t), Y(t))$ be a random process satisfying (6.4), where the distribution of the $\left\{X_{i}\right\}$ is given by a CDF $F$, with the corresponding $S F \bar{F}$, and for each $n \in \mathbb{N},\left(X_{i}^{(n)}\right)_{i \in \mathbb{N}} \stackrel{d}{=}\left(X_{i}\right)_{i \in \mathbb{N}}$. Then we have the following:

(i) For $t \in \mathbb{R}_{+}$, the SF of $X(t)$ is given by $\bar{F}^{t}$ while the CDF of $Y(t)$ is given by $F^{t}$.

(ii) For $t \in \mathbb{R}_{+}$, the joint $C D F$ of $(X(t), Y(t))$ is given by (3.12).

(iii) Both, $X(t)$ and $Y(t)$ are a pure jump processes, with right-hand-side continuous trajectories. Moreover, the trajectories of $X(t)$ are non-increasing, while the trajectories of $Y(t)$ are non-decreasing.

(iv) If $t=n \in \mathbb{N}$, then $X(n)=\bigwedge_{k=1}^{n} X_{k}$ and $Y(n)=\bigvee_{k=1}^{n} X_{k}$ with probability one.

(v) If $t_{n} \in(n, n+1)$ is the location of the last jump of $X$ on $(n, n+1)$, then $X$ on $\left(n, t_{n}\right)$ is independent of $X(t)$ on $\mathbb{R}^{+} \backslash\left(n, t_{n}\right)$. Similar property holds for $Y(t)$.

Observe that Eq. 6.4 does not define the process $(X(t), Y(t))$ uniquely. In the following remarks, we note two important special cases.

Remark 6.2 The max-min process is defined in a similar fashion to the extremal process by taking the sequences $\left(X_{i}^{(n)}\right)_{i \in \mathbb{N}}$, which are mutually independent across $n \in \mathbb{N}_{0}$, and $X_{j}=X_{1}^{(j-1)}$. Also, the Sibuya processes $N^{(n)}$ are assumed mutually independent across $n \in \mathbb{N}_{0}$.

Remark 6.3 Another process, which will be referred to as the record process, is defined by taking a single sequence $\left(X_{i}\right)_{i \in \mathbb{N}}$ and setting $\left(X_{i}^{(n)}\right)_{i \in \mathbb{N}}=\left(X_{i+n}\right)_{i \in \mathbb{N}}$. Let us note that, in contrast with the extremal processes described in Resnick (1987), here $X(t)$ and $Y(t)$ are directly related to the maxima and the minima of the underlying sequence $\left\{X_{n}\right\}$. Moreover, if the value of $X_{n}(n>1)$ does not set a new record, so that $S_{n-1} \leq X_{n} \leq M_{n-1}$, the trajectories of $X(t)$ and $Y(t)$ are constant on the interval $[n-1, n]$. If $X_{n}$ does set a new record with $X_{n}>M_{n-1}$, then the trajectory of $X(t)$ is still constant (and equal to $S_{n-1}$ ) on $[n-1, n]$ while that of $Y(t)$ will be increasing from $Y(n-1)=M_{n-1}$ to $Y(n)=M_{n}$ through a sequence of jumps on the interval $[n-1, n]$, with both, the jump sizes and their locations, affected by the "future" values of the $\left\{X_{i}\right\}$. Similarly, if $X_{n}$ sets a new downward record with $X_{n}<S_{n-1}$, then the trajectory of $Y(t)$ will be constant (and equal to $M_{n-1}$ ) on the interval $[n-1, n]$ while that of $X(t)$ will be decreasing from $X(n-1)=S_{n-1}$ to $X(n)=S_{n}$ through a sequence of jumps on $[n-1, n]$, related to the future values of $\mathbf{X}$. Interestingly, if a trajectory of either component of our process does go up (or down) in $(n-1, n)$, one can predict a record value is to occur at time $t=n$. 
While complete analysis of the bivariate process with the structure given in Eq. 6.4 is beyond the scope of this paper, below we provide few additional remarks shedding more light on its basic properties. Let us start with a discussion of the structure of the Sibuya process $N(t)$, discussed fully in (Kozubowski and Podgórski 2017). Its jump sizes (viewed from right to left) can be iteratively computed from the following scheme that is based on Eq. 5.4 and on the fact that a generalized Sibuya distribution $G S_{1}(t, r)$ can be obtained from

$$
S \stackrel{d}{=}\left[\frac{\log (U)}{\log (1-B)}\right]+1
$$

where $U$ has uniform distribution and is independent of $B$, which has beta distribution with parameters $t$ and $1-t+r$, see Proposition 9 in (Kozubowski and Podgórski 2017). We consider simulation of $N$ jumps. We take $\tau_{N}=1$ as the first value of the jump at $t_{N}=1$, and then recursively evaluate the jumps at the locations $t_{N-k}=$ $U_{k} t_{N-k+1}, k \geq 1$, by taking the subsequent jumps $\tau_{N-k}=\left[\log \left(V_{k}\right) / \log \left(1-p_{k}\right)\right]+1$, where the $\left\{V_{k}\right\}$ are IID uniformly distributed (independently of everything else), while the $p_{k}$ 's are sampled independently from beta distribution with parameters $\left(t_{N-k+1}, 1-t_{N-k+1}+\tau_{N-k+1}\right)$. Due to large values that the process $N(t)$ takes in the vicinity of zero, this scheme is much more effective in simulating values of the Sibuya process than directly from Eq. 5.1, which would require an enormous number of uniform variables to be simulated. Nevertheless, typically there are only very few jumps that can be realistically observed in the process due to their extremely large values when process nears in the argument to zero. This can be seen from Fig. 4, where twenty samples from the process are given on the logarithmic scale in values. By having a way to simulate the Sibuya process on can have the max-min process on the half-line obtained effectively from the following result.

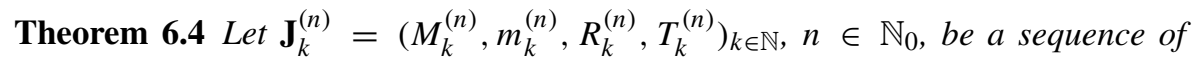
independent Markov jump processes with distribution described in Theorem 5.1. The max-min process described in Remark 6.2 admits the following representation:

$$
\left.(X(t), Y(t)) \stackrel{d}{=} \sum_{k=1}^{\infty}\left(\bigwedge_{j=1}^{\left[t^{-}\right]} X_{1}^{(j-1)} \wedge M_{k}^{\left(\left[t^{-}\right]\right)}, \bigvee_{j=1}^{\left[t^{-}\right]} X_{1}^{(j-1)} \vee m_{k}^{\left(\left[t^{-}\right]\right)}\right) \mathbb{I}_{\left(T_{k}^{\left(\left[t^{-}\right]\right)}, T_{k-1}^{\left(\left[t^{-}\right]\right)}\right]}(\langle t\rangle)\right)
$$

The proof of the result is not included since it follows easily from Theorem 5.1 and the definition of the max-min process..

\section{Proofs}

Proof of Lemma 2.1 This result follows from a standard conditioning argument. Since

$$
\mathbb{P}\left(\bigvee_{j=1}^{N} X_{j} \leq x\right)=\sum_{n=1}^{\infty} \mathbb{P}\left(\bigvee_{j=1}^{n} X_{j} \leq x\right) p_{n}=\sum_{n=1}^{\infty} F^{n}(x) p_{n},
$$


we obtain $F_{X}(x)=G_{N}(F(x))$. The analogous argument leads to $\bar{F}_{Y}(y)=$ $G_{N}(\bar{F}(y))$.

Proof of Lemma 3.1 Write $H(x, y)=\mathbb{P}(X \leq x)-\mathbb{P}(X \leq x, Y>y)$, which, by Lemma 2.1, leads to $H(x, y)=G_{N}(F(x))-\mathbb{P}(X \leq x, Y>y)$. Next, we consider the term $\mathbb{P}(X \leq x, Y>y)$. Clearly, this reduces to zero whenever $x \leq y$ (since, by the definition of these variables, we have $X \geq Y$ ). In turn, for $x>y$, by conditioning on $N$ we obtain

$$
\mathbb{P}(X \leq x, Y>y)=\sum_{n=1}^{\infty} \mathbb{P}\left(\bigvee_{j=1}^{N} X_{j} \leq x, \bigwedge_{j=1}^{N} X_{j}>y \mid N=n\right) \mathbb{P}(N=n) .
$$

Since the $\left\{X_{i}\right\}$ and $N$ are independent, we have

$$
P\left(\bigvee_{j=1}^{N} X_{j} \leq x, \bigwedge_{j=1}^{N} X_{j}>y \mid N=n\right)=P\left(\bigvee_{j=1}^{n} X_{j} \leq x, \bigwedge_{j=1}^{n} X_{j}>y\right)=\left[\mathbb{P}\left(y<X_{j} \leq x\right)\right]^{n},
$$

so that

$$
\mathbb{P}(X \leq x, Y>y)=\sum_{n=1}^{\infty}(F(x)-F(y))^{n} \mathbb{P}(N=n)=G_{N}(F(x)-F(y)) .
$$

This completes the proof.

Proof of Proposition 3.6 First, assume that $t \in(0,1]$, so that $\left[t^{-}\right]=0$ and the representation (3.11) coincides with Eq. 3.3 with $\alpha=\langle t\rangle$ (as the min and the max over an empty set are $\infty$ and $-\infty$, respectively). In this case the result follows from Corollary 3.2, since the CDF (3.12) coincides with Eq. 3.4. Next, assume that $t>1$, and write $n=\left[t^{-}\right] \in \mathbb{N}$ and $\alpha=\langle t\rangle \in(0,1]$. Further, denote

$$
(\tilde{X}, \tilde{Y})=\left(\bigwedge_{j=1}^{n} X_{j}, \bigvee_{j=1}^{n} X_{j}\right) \text { and }\left(X_{\alpha}, Y_{\alpha}\right)=\left(\bigvee_{j=n+1}^{n+N_{\alpha}} X_{j}, \bigwedge_{j=n+1}^{n+N_{\alpha}} X_{j}\right),
$$

noting that $(X, Y)=\left(\tilde{X} \wedge X_{\alpha}, \tilde{Y} \vee Y_{\alpha}\right)$ and the two random vectors above are independent. Using this representation allows for expressing the joint $\mathrm{CDF}$ of $(X, Y)$ as

$$
H(x, y)=\mathbb{P}\left(\tilde{Y} \vee Y_{\alpha} \leq y\right)-\mathbb{P}\left(\tilde{X} \wedge X_{\alpha}>x, \tilde{Y} \vee Y_{\alpha} \leq y\right) .
$$

By independence of $\tilde{Y}$ and $Y_{\alpha}$, the first term on the right-hand-side above can be written as

$$
\mathbb{P}\left(\tilde{Y} \vee Y_{\alpha} \leq y\right)=\mathbb{P}\left(\tilde{Y} \leq y, Y_{\alpha} \leq y\right)=F^{n+\alpha}(y),
$$

since the marginal CDFs of $\tilde{Y}$ and $Y_{\alpha}$ are $F^{n}$ and $F^{\alpha}$, respectively. Next, we write the second term on the right-hand-side in Eq. 7.1 as

$$
\mathbb{P}\left(\tilde{X}>x, X_{\alpha}>x, \tilde{Y} \leq y, Y_{\alpha} \leq y\right)=\mathbb{P}(\tilde{X}>x, \tilde{Y} \leq y) \mathbb{P}\left(X_{\alpha}>x, Y_{\alpha} \leq y\right),
$$


which is equivalent to

$$
(\mathbb{P}(\tilde{Y} \leq y)-\mathbb{P}(\tilde{X} \leq x, \tilde{Y} \leq y))\left(\mathbb{P}\left(Y_{\alpha} \leq y\right)-\mathbb{P}\left(X_{\alpha} \leq x, Y_{\alpha} \leq y\right)\right) .
$$

To complete the proof, substitute the CDFs of $(\tilde{X}, \tilde{Y})$ and $\left(X_{\alpha}, Y_{\alpha}\right)$, which are given by Eqs. 1.3 and 3.4, respectively.

Proof of Theorem 5.1 The max-min process can only jump at the locations of jumps of $N(t)$ that are represented in Eq. 5.4. Thus, clearly

$$
(X(t), Y(t)) \stackrel{d}{=} \sum_{k=1}^{\infty}\left(\bigvee_{j=1}^{R_{k}} X_{j}, \bigwedge_{j=1}^{R_{k}} X_{j}\right) \mathbb{I}_{\left(T_{k}, T_{k-1}\right]}(t) .
$$

Now, the bivariate jumps can be written as follows:

$$
\begin{aligned}
\left(M_{k}, m_{k}\right) & =\left(\bigvee_{j=1}^{R_{k}} X_{j}, \bigwedge_{j=1}^{R_{k}} X_{j}\right) \\
& =\left(\begin{array}{c}
M_{k-1} \vee \bigvee_{j=R_{k-1}+1}^{R_{k}} X_{j}, m_{k-1} \wedge \bigwedge_{j=R_{k-1}+1}^{R_{k}} X_{j}
\end{array}\right) \\
& =\left(\bigvee_{j=R_{k-1}+1}^{R_{k-1}+S_{k}} X_{j}, m_{k-1} \wedge \bigwedge_{j=R_{k-1}+1}^{R_{k-1}+S_{k}} X_{j}\right),
\end{aligned}
$$

where the $\left\{S_{k}\right\}$, conditionally on $R_{k-1}=r$ and $T_{k-1}=t$, have generalized Sibuya distribution $G_{1}(t, r)$ as presented in Eq. 5.4. This, conditionally on $\left(M_{k-1}, m_{k-1}, R_{k-1}, T_{k-1}\right)=(M, m, r, t)$, yields

$$
\left(M_{k}, m_{k}\right) \stackrel{d}{=}\left(M \vee W_{k}, m \wedge w_{k}\right),
$$

where

$$
\left(W_{k}, w_{k}\right) \stackrel{d}{=}\left(\bigvee_{j=1}^{S_{k}} X_{j}, \bigwedge_{j=1}^{S_{k}} X_{j}\right) .
$$

The final distributional form of $\left(W_{k}, w_{k}\right)$ follows from Lemma 3.1 applied to the case of $N$ with the generalized Sibuya PMF given in Eq. 5.3.

Proof of Proposition 5.3 It follows from the proof of Theorem 5.1 that

$$
\left(W_{k}, w_{k}\right) \stackrel{d}{=}\left(\bigvee_{i=1}^{S_{k}} X_{i}, \bigwedge_{i=1}^{S_{k}} X_{i}\right)
$$


where the $\left\{X_{i}\right\}$ are IID with distribution given by the CDF $F$. From the well known representation of the order statistics, the largest and the smallest order statistics can be expressed by the arrivals $\Gamma_{i}$ of a standard Poisson process as

$$
\left(\bigvee_{i=1}^{S_{k}} X_{i}, \bigwedge_{i=1}^{S_{k}} X_{i}\right) \stackrel{d}{=}\left(F^{-1}\left(\frac{\Gamma_{S_{k}}}{\Gamma_{S_{k}+1}}\right), F^{-1}\left(\frac{\Gamma_{1}}{\Gamma_{S_{k}+1}}\right)\right),
$$

and the conclusion follows.

Proof of Proposition 5.5 Suppose that for some deterministic $c_{p}>0$ we have convergence in distribution to some (necessarily non-negative) random variable $Z$ stated in the proposition. Expressing this in terms of Laplace transforms (LTs) we will have

$$
\mathbb{E}\left(e^{-t c_{p} N_{p}}\right)=G_{p}\left(e^{-t c_{p}}\right) \rightarrow \psi(t) \text { as } p \rightarrow 0
$$

for each $t>0$, where $\psi(\cdot)$ is the LT of $Z$ and $G_{p}(s)$ is the PGF of $N_{p}$ given by the right-hand-side of Eq. 2.2 with $\alpha=p$. Consequently, we conclude that, for each $t>0$, we would have

$$
\left(1-e^{-t c_{p}}\right)^{p} \rightarrow 1-\psi(t) \text { as } p \rightarrow 0 .
$$

However, we have

$$
\left(1-e^{-t c_{p}}\right)^{p}=\left(\frac{1-e^{-t c_{p}}}{c_{p}} c_{p}\right)^{p}=\left(\frac{1-e^{-t c_{p}}}{c_{p}}\right)^{p} c_{p}^{p}
$$

and, since we must have $c_{p} \rightarrow 0$ as $p \rightarrow 0$, we would have

$$
\left(\frac{1-e^{-t c_{p}}}{c_{p}}\right)^{p} \rightarrow t^{0}=1 \text { as } p \rightarrow 0
$$

In view of this, along with Eqs. 7.3-7.4, we conclude that, for each $t>0$, we must have the convergence

$$
c_{p}^{p} \rightarrow 1-\psi(t) \text { as } p \rightarrow 0,
$$

which can not hold unless the right-hand-side is independent on $t$, which is the case only if $\mathbb{P}(Z=0)=1$. This concludes the proof.

Proof of Theorem 6.1 Let $Y_{1}(t)=\bigvee_{k=1}^{\left[t^{-}\right]} X_{k}$ and $Y_{2}(t)=\bigwedge_{i=1}^{N^{\left[t^{-}\right]}(\langle t\rangle)} X_{\left[t^{-}\right]+i}$. It is clear that $Y_{1}(t)$ and $Y_{2}(t)$ are independent for each fixed $t$, so that, by Corollary 2.3,

$$
\mathbb{P}(Y(t) \leq x)=\mathbb{P}\left(Y_{1}(t) \leq x\right) \mathbb{P}\left(Y_{2}(t) \leq x\right)=F^{\left[t^{-}\right]}(x) \cdot F^{\langle t\rangle}(x)=F^{t}(x) .
$$

Both $Y_{1}$ and $Y_{2}$ are non-decreasing with right hand side continuous trajectories.

Acknowledgments The authors thank two referees for their remarks that fundamentally improved the original version. The research of the first author was partially funded by the European Union's Seventh Framework Programme for research, technological development and demonstration under grant agreement no 318984 - RARE and the research of the second author was partially supported by Riksbankens Jubileumsfond Grant Dnr: P13-1024:1 and Swedish Research Council Grant Dnr: 2013-5180. 
Open Access This article is distributed under the terms of the Creative Commons Attribution 4.0 International License (http://creativecommons.org/licenses/by/4.0/), which permits unrestricted use, distribution, and reproduction in any medium, provided you give appropriate credit to the original author(s) and the source, provide a link to the Creative Commons license, and indicate if changes were made.

\section{Appendix}

In the following algorithm in $\mathbf{R}$ language random samples from the copula given in Proposition 3.8 are simulated. In it, first, it is decided whether a random value is from the singular or the absolutely continuous component. Then, in the continuous case, a random variate $V$ is simulated from its marginal (3.19) and then a random variate $U$, conditionally on the obtained value $V$, is simulated from the conditional distribution (3.21).

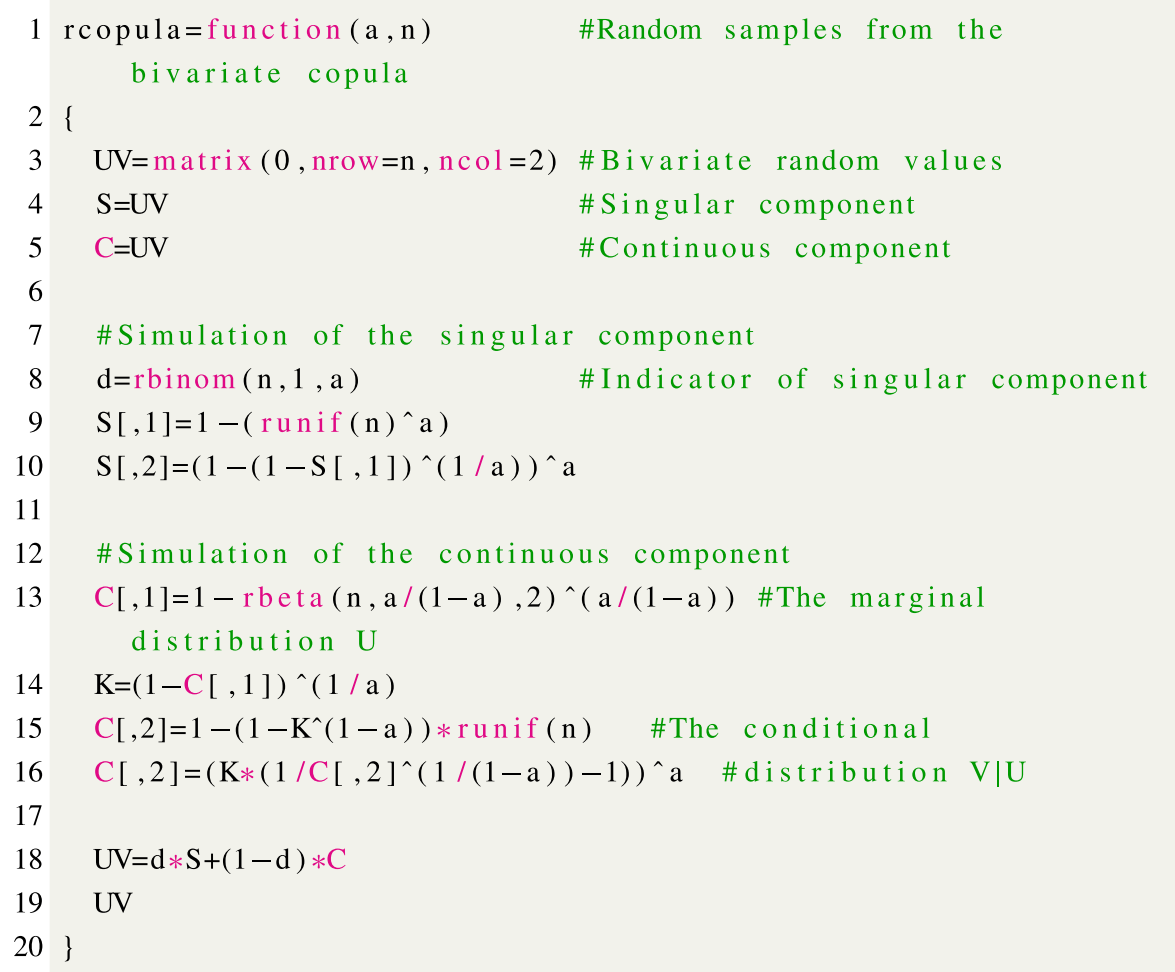

\section{References}

Arnold, B., Balakrishnan, N., Nagaraja, H.: A First Course in Order Statistics. Wiley, New York (1992) Avérous, J., Genest, C., Kocha, S.: On the dependence structure of order statistics. J. Multivariate Anal. 94, 159-171 (2005) 
Beirlant, J., Teugels, J., Vynckier, P.: Practical Analysis of Extreme Values. Leuven University Press, Leuven (1996)

Castillo, E.: Extreme Value Theory in Engineering. Academic Press, San Diego (1988)

Christoph, G., Schreiber, K.: Discrete stable random variables. Statist. Probab. Lett. 37, 243-247 (1998)

Christoph, G., Schreiber, K.: Shifted and scaled Sibuya distribution and discrete self-decomposability. Statist. Probab. Lett. 48(2), 181-187 (2000)

David, H.: Order Statistics, 2nd edn. Wiley, New York (1981)

Devroye, L.: A triptych of discrete distributions related to the stable law. Statist. Probab. Lett. 18, 349-351 (1993)

Dwass, M.: Extremal process. Ann. Math Statist. 35, 1718-1725 (1964)

Dwass, M.: Extremal processes, ii. Ill. J. Math. 10, 381-391 (1966)

Embrechts, P., Klüppelberg, C., Mikosch, T.: Modelling Extremal Events for Insurance and Finance. Springer, Berlin (1997)

Galambos, J.: The Asymptotic Theory of Extreme Order Statistics, 2nd edn. Wiley, New York (1987)

Gompertz, B.: On the nature of the function expressive of the law of human mortality, and on the new mode of determining the value of life contingencies. Phil. Trans. Roy. Soc. London 115, 513-585 (1825)

Gumbel, E.: Statistics of Extremes. Columbia University Press, New York (1958)

Gupta, R., Gupta, P., Gupta, R.: Modeling failure time data by the Lehman alternatives. Comm. Statist. Theory Methods 27, 887-904 (1998)

Gupta, R., Kundu, D.: Generalized exponential distributions. Austral. \& New Zealand J. Statist. 41(2), 173-188 (1999)

Gupta, R., Kundu, D.: Generalized exponential distribution: existing results and some recent developments. J. Statist. Plann. Inference 137, 3537-3547 (2007)

Harter, H.: A bibliography of extreme-value theory. Internat. Statist. Rev. 46, 279-306 (1978)

Kotz, S., Nadarajah, S.: Extreme Value Distributions: Theory and Applications. Imperial College Press, London (2000)

Kozubowski, T., Podgórski, K.: A generalized Sibuya distribution. Ann. Inst. Statist. Math. https://doi.org/10.1007/s10463-017-0611-3 (2017)

Kozubowski, T., Podgórski, K.: Transmuted distributions and random extrema. Statist. Probab. Lett. 116, 6-8 (2016)

Lamperti, J.: On extreme order statistics. Ann. Math Stat. 35(4), 1726-1737 (1964)

Leadbetter, M., Lindgren, G., Rootzén, H.: Extremes and Related Properties of Random Sequences and Processes. Springer, Berlin (1983)

Marshall, A., Olkin, I.: A new method for adding a parameter to a family of distributions with application to the exponential and Weibull families. Biometrika 85(3), 641-652 (1997)

Mudholkar, G., Srivastava, D.: Exponentiated Weibull family for analyzing bathtub failure data. IEEE Trans. Reliability 42, 299-302 (1993)

Mudholkar, G., Srivastava, D., Freimer, M.: The exponentiated Weibull family: a reanalysis of the bus motor failure data. Technometrics 37, 436-445 (1995)

Nelsen, R.: An Introduction to Copulas. Springer, New York (1999)

Pakes, A.: Characterization of discrete laws via mixed sums and Markov branching processes. Stochastic Proc. Appl. 55, 285-300 (1995)

Pal, M., Ali, M., Woo, J.: Exponentiated Weibull distribution. Statistica (Bologna) 66(2), 139-147 (2006)

Pillai, R., Jayakumar, K.: Discrete Mittag-Leffler distributions. Statist. Probab. Lett. 23, 271-274 (1995)

Reiss, R.-D., Thomas, M.: Statistical Analysis of Extreme Values with Applications to Insurance, Finance, Hydrology and Other Fields. Basel, Birkhaüser (2001)

Resnick, S.: Extreme Values, Regular Variation, and Point Processes. Springer, New York (1987)

Resnick, S., Rubinovitch, M.: The structure of extremal processes. Adv. Appl. Prob. 5, 287-307 (1973)

Schmitz, V.: Revealing the dependence structure between $x_{(1)}$ and $x_{(n)}$. J. Statist. Plann. Inference 123, 41-47 (2004)

Shaw, W., Buckley, I.: The alchemy of probability distributions: beyond Gram-Charlier expansions, and a skew-kurtotic-normal distribution from a rank transmutation map. arXiv:0901.0434v1 [q-fin.ST] (2009)

Sibuya, M.: Generalized hypergeometric, digamma, and trigamma ditributions. Ann. Inst. Statist. Math. 31, 373-390 (1979)

Sklar, A.: Fonctions de répartition á n dimensions et leurs marges. Publ. Inst. Statist. Univ. Paris 8, 229-231 (1959) 